\begin{tabular}{|c|l|}
\hline Title & Communication Technologies and Spatial Organization of Multi-unit Firms in Metropolitan A reas \\
\hline Author(s) & Ota, Mitsuru; Fujita, Masahisa \\
\hline Citation & $\begin{array}{l}\text { Environmental science, Hokkaido University : journal of the Graduate School of Environmental Science, Hokkaido } \\
\text { University, Sapporo, 13(2), 45-73 }\end{array}$ \\
\hline Issue Date & 1991-03-30 \\
\hline Doc URL & http://hdl.handle.net/2115/37260 \\
\hline Type & bulletin (article) \\
\hline File Information & 13(2)_45-73.pdf \\
\hline
\end{tabular}

Instructions for use 


\begin{tabular}{|l|l|l|l|}
\hline Environ. Sci., Hokkaido University & $13(2)$ & $45 \sim 73$ & Dec. 1990 \\
\hline
\end{tabular}

\title{
Communication Technologies and Spatial Organization of Multi-unit Firms in Metropolitan Areas
}

\author{
Mitsuru Ota* and Masahisa Fujita** \\ *Department of Regional Planning, Division of Environmental Planning \\ Graduate School of Environmental Science \\ Hokkaido University, Sapporo, 060, Japan \\ **Department of Regional Science, University of Pennsylvania \\ Philadelphia, PA 19104-6209, U.S.A.
}

\begin{abstract}
Departing from traditional location theory (which treats a firm as a single-unit entity), in this paper we consider that each firm consists of multiple units which exchange information or services. Specifically, we develop a general equilibrium model of the city, in which each firm consists of a front-unit (e.g., business office) and back-unit (e.g., plant or back-office). Each front-unit interacts with all other frontunits for the purpose of business communications, while each back-unit exchanges information or management services only with the front-unit of the same firm. Each firm must choose the location of its front-unit and back-unit optimally. The equilibrium spatial configuration of the city is determined as an outcome of interactions among all firms and households through competitive land and labor markets. We show that depending on parameters, a variety of interesting patterns of metropolitan spatial organization emerges.
\end{abstract}

\section{Introduction}

In the traditional location theory, the firm has been treated as a single-unit entity. ${ }^{1}$ In the context of urban location theory, this single-unit approach to a firm location might have been appropriate because, in the past, most firms in a city conducted their main activities at a single location. Recently, however, many firms in large cities have started to conduct their activities at separate locations.

For example, many business firms (e.g., investment banks) in large cities of developed countries have recently moved a part of their office activities (such as billing, bookkeeping, planning, research, and employee training) to the suburbs. A business firm typically conducts a part of its activities (such as face-to-face communication with other business firms) at the front-office located in the central business district (CBD), and the rest of its activities are carried out at the back-office located in the suburbs. As another example, many manufacturing firms in large cities conduct their business activities at their headquarters located in the CBD while their manufacturing plants remain in the suburbs. This spatial segregation of firm activities is mainly due to the recent development of telecommunication technologies (including computer related communication techno- 
logies)..$^{2}$

If a firm is located and conducts all of its activities in the CBD, both inter-communications (among its various units) and extra-communications (with other business offices) can be maintained most efficiently. However, by doing so, the firm must incur large floor-rents, while its workers must commute over long distances from the suburbs. On the other hand, if the firm moves all of its activities to the suburbs, it will incur a severe disadvantage in (face-to-face) communications with other business offices. Therefore, given recent developments in telecommunication technologies, a typical large firm (e.g., Merrill Lynch, the largest investment bank in the U.S.) may keep its front-office in the CBD (e.g., Manhattan) and build a spacious back-office (or, production plant) in the far removed suburbs (e.g., Princeton), with the two offices connected by modern communication equipment such as inter-computer networks and facsimiles.

Given these innovations in the spatial reorganization of firm activities, in this paper we develop a multi-unit approach to the location of firms, and examine possible impacts of communication technologies on the spatial organization of large cities. Specifically, we develop a general equilibrium model of the city, in which each firm consists of a front-unit (e.g., business office) and back-unit (e.g., plant, back-office, or R \& D unit). Each front-unit is assumed to interact with all other front-units for the purpose of business communications, while each back-unit exchanges information or management services only with the front-unit of the same firm. Each firm must choose the location of its front-unit and backunit optimally. The equilibrium spatial configuration of the city is determined as an outcome of interactions among all front-units, back-units and households (i.e., workers) through competitive land and labor markets. Depending on parameter specifications (e.g., communication costs among front-units, communication costs between front-units and back-units, and commuting costs of workers), a variety of interesting patterns of metropolitan spatial organization emerges.

In section 2, we develop the model. Although our model is a multi-unit extension of the nonmonocentric city model by Ogawa and Fujita (1980), the two models are different in an essential aspect. That is, as was noted before, in our model each firm may occupy land at two different locations. This makes it difficult to apply the traditional concept of bid rent functions. We overcome this difficulty by reformulating the firm's behavior in a decentralized form. That is, in section 3 , we introduce shadow profit functions of front -units and back-units respectively, and propose to obtain the optimal location-pair (i.e., location of the front-unit and back-unit) of each firm as a Nash location-equilibrium in which each front-unit (back-unit) chooses its location optimally (so as to maximize its own shadow profit) while taking the location of the associated back-unit (front-unit) as given.

In section 4, first we describe the conditions for a Nash equilibrium configuration of the city, in which each front-unit and back-unit maximizes its own shadow profit function. A Nash equilibrium configuration, however, does not always represent a global equilibrium configuration in which each firm maximizes its (real) profit function by choosing the location of its front-unit and back-unit simultaneously. Hence, by introducing an additional requirement, we obtain the global equilibrium conditions. We also introduce further specifications of several parameter functions. 
It turns out that given each set of parameters, although there may exist more than one Nash equilibrium configuration, there exists one and only one global equilibrium configuration. In section 5, we show that there exists, in total, eleven different (global) equilibrium configurations. We discuss which configuration is likely to be observed under what conditions. Finally, in section 6 , we discuss further research directions.

\section{The model}

Assume that a city develops in a given geographical space $X$. The city accommodates a continuum of homogeneous firms and a continuum of homogeneous households (i.e., workers). Each firm consists of two units, the front-unit and back-unit. Each front-unit interacts with all other front-units for the purpose of business communications, while each back-unit is assumed to interact (for exchange of information or management services) with only the front-unit of the same firm. ${ }^{3}$ Each firm must choose the location of its frontunit and back-unit optimally. Households supply labor to firms by commuting, and receive wages. We assume that all land in the city is owned by absentee landlords, and that the land not occupied by households or firms is used for agriculture, yielding a constant rent $R_{A}$. Everywhere in $X$, the density of land is assumed to be 1 . Furthermore, both land and labor markets are assumed to be perfectly competitive. The equilibrium spatial configura. tion of the city is determined as an outcome of interactions among all firms and households through competitive land and labor markets. We describe below the behavior of each household and firm in detail.

\subsection{Households}

There are $N$ identical households in a city. The utility function of each household is given by $U(S, Z)$, where $S$ represents the consumption of land and $Z$ the consumption of the composite (consumer) goods. For simplicity, we assume that $S$ is fixed for all households at some constant $S_{h}$. We also assume that each household supplies a unit of labor to a firm, and that the composite goods are imported at a constant price of 1 . Then, if a household chooses to reside at $x \in X$ and work at $x_{w} \in X$, then its budget constraint is given by

$$
Z+R(x) S_{h}+T\left(x, x_{w}\right)=W\left(x_{w}\right)
$$

where $T\left(x, x_{w}\right)$ is the commuting cost from $x$ to $x_{w}$, and $W\left(x_{w}\right)$ is the wage rate at $x_{w}$. Now, since a lot size is fixed at the constant $S_{h}$, the objective of each household is to choose a residential location $x$ and working location $x_{w}$ which maximize the amount of compositegoods consumption given by

$$
Z\left(x, x_{w}\right)=W\left(x_{w}\right)-R(x) S_{h}-T\left(x, x_{w}\right) .
$$




\subsection{Firms}

There are $M$ homogeneous firms in the city. ${ }^{4}$ Each firm produces some type of service or goods which is exported outside the city at the constant (normalized) price of 1 . As noted before, each firm consists of a front-unit and back-unit. The main task of the front-unit of a firm is to communicate with all other firms (through their front-units). The back-unit of a firm engages in the internal operation of the firm, and makes contact with the frontunit of the same firm only. The relationship between front-units and back-units are depicted in Figure 1. If a firm chooses its front-unit at $x \in X$ and back-unit at $y \in X$, the

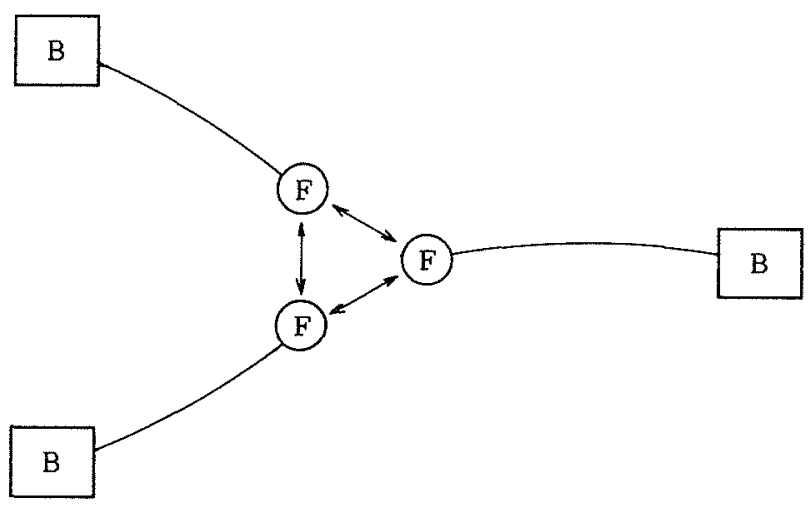

Figure 1. Front-units (F) and Back-units (B).

firm incurs an intrafirm communication cost, $\Gamma(x, y)$, per unit of time. Here, we assume that such intrafirm communications can be attained through routine contacts between the two units, and hence the cost $\Gamma(x, y)$ depends only on the location pair, $x$ and $y$. It is assumed that $\Gamma(x, x)=0$ for all $x \in X$.

Each front-unit, however, actively engages in communication with other front-units. Here, it is postulated that communication itself is measurable in terms of some level of contact activity, $q$ (e.g., the number of face-to-face contacts per unit of time), and that each front-unit is free to choose its optimal level of contact activity with other front-units. ${ }^{5}$

Each front-unit is assumed to require $S_{f}$ units of land and $L_{f}$ units of labor, while each back-unit reguires $S_{b}$ units of land and $L_{b}$ units of labor. [Here, each $S_{f}, L_{f}, S_{b}$, and $L_{b}$ is a given positive constant.] Let $f(x)$ and $b(x)$ denote respectively the density of front-units and that of back-units at each location $x \in X$, and $R(x)$ and $W(x)$ represent respectively the land rent and wage rate at each $x$. We assume that if a firm having the front-unit at $x$ and back-unit at $y$ chooses a level, $q(x, z)$, of communication with every single front-unit (of other firms) at location $z$ (for all $z \in X$ ), the profit $\Pi$ of the firm (per unit of time) may be given by 


$$
\begin{aligned}
\Pi(x, y, q(x, \cdot))= & \int_{X} V[q(x, z)] f(x) d z-\int_{X} c(x, z) q(x, z) f(z) d z \\
& -R(x) S_{f}-W(x) L_{f}-R(y) S_{b}-W(y) L_{b}-\Gamma(x, y) .
\end{aligned}
$$

Here, $V$ is an appropriate numerical function and $V[q(x, z)]$ represents the contribution of contacts $q(x, z)$ (with a front-unit at $z$ ) to the revenue of the firm, and $c(x, z)$ represents the cost of communication per unit contact between locations $x$ and $z .^{6}$ Hence, on the right side of (2.3), the first and second terms represent respectively the total benefits and total costs of interfirm communications, the third and fourth terms represent the land cost and labor cost of the front-unit, the fifth and sixth terms those of the back-unit, and the last term represents the intrafirm communication cost.

Each firm chooses its front-unit location $x$, back-unit location $y$, and contact pattern $q(x, \cdot)$ so as to maximize the profit $\Pi(x, y, q(x, \cdot))$ [taking front-unit distribution $f(y)$ and other market variables as given]. To simplify expression (2.3), note that the first two terms on the right side of (2.3) can be rewritten as

$$
\begin{aligned}
\int_{X} V[q(x, z)] f(z) d z & -\int_{X} c(x, z) q(x, z) f(x) d z \\
& =\int_{X}\{V[q(x, z)]-c(x, z) q(x, z)\} f(z) d z
\end{aligned}
$$

Therefore, for each pair of $x$ and $z \in X$, the optimal $q(x, z)$ can be determined independent of $f$. Namely, for each $c \in \mathfrak{N}_{+}$, let $q^{*}(c)$ be the solution to the following maximization problem:

$$
\max _{q} V(q)-c q \quad \text { subject to } q \geq 0 \text {. }
$$

Then, the optimal $q(x, z)$ can be expressed as $q^{*}[c(x, y)]$. Hence, if we define the accessibility measure, $a(c)$, for each $c \in \mathfrak{N}_{+}$by

$$
a(c) \equiv V\left[q^{*}(c)\right]-c q^{*}(c),
$$

then for each location pair, $x$ and $y$, the maximum profit $\Pi(x, y) \equiv \Pi\left(x, y, q^{*}[c(x, \cdot)]\right.$ is given $\mathrm{by}^{7}$

$$
\Pi(x, y)=A(x)-R(x) S_{f}-W(x) L_{f}-R(y) S_{b}-W(y) L_{b}-\Gamma(x, y)
$$

where

$$
A(x)=\int_{X} a[c(x, z)] f(z) d z .
$$

In (2.7), $A(x)$ represents the aggregate accessibility measure of each location $x \in X$.

From (2.6), given distributions $A(\cdot), R(\cdot)$, and $W(\cdot)$, each firm chooses its front-unit location $x$ and back-unit location $y$ so as to maximize its profit $\Pi(x, y)$. While, from (2. 2 ), each household chooses a residential location $x$ and working location $x_{w}$ so as to maximize the composite-goods consumption $Z\left(x, x_{w}\right)$. An equilibrium is reached when all $M$ firms achieve the same maximum profit, all $N$ households the same maximum utility, and land and labor markets are cleared everywhere. Here, to assure that a full employ. ment prevails in the city at equilibrium, we assume that

$$
N=\left(L_{f}+L_{b}\right) M
$$




\section{Reformulation of the firm behavior in a decentralized form}

We wish to express the equilibrium conditions of the land-use model above by using the concept of bid rent functions. Our model, however, is fundamentally different from traditional models in one respect. Namely, as shown by (2.6), each firm occupies land at two different locations (provided $x \neq y$ ). ${ }^{8}$ This makes it difficult to apply the traditional concept of bid rent functions.

One way to overcome this difficulty, which we adopt in this paper, is to reformulate the firm's behavior in a decentralized form. Namely, assuming that we can find a (shadow) profit function, $\Pi_{f}(x \mid y)$, of each front-unit and a (shadow) profit function, $\Pi_{b}(y \mid x)$, of each back-unit such that

$$
\Pi\left(x^{*}, y^{*}\right)=\max _{x \in X, y \in X} \Pi(x, y) \Rightarrow\left\{\begin{array}{l}
\Pi_{f}\left(x^{*} \mid y^{*}\right)=\max _{x \in X} \Pi_{f}\left(x \mid y^{*}\right) \\
\Pi_{b}\left(y^{*} \mid x^{*}\right)=\max _{y \in X} \Pi_{b}\left(y \mid x^{*}\right),
\end{array}\right.
$$

and

$$
\left.\begin{array}{l}
\Pi_{f}\left(x^{*} \mid y^{*}\right)=\max _{x \in X} \Pi_{f}\left(x \mid y^{*}\right) \\
\Pi_{b}\left(y^{*} \mid x^{*}\right)=\max _{y \in X} \Pi_{b}\left(y \mid x^{*}\right)
\end{array}\right\} \Rightarrow \Pi\left(x^{*}, y^{*}\right)=\max _{x \in X, y \in X} \Pi(x, y) .
$$

Condition (3.1) means that if $\left(x^{*}, y^{*}\right)$ is an optimal location-pair of a firm, then it is also a Nash equilibrium location-pair for the front-unit and back-unit, where each unit maximizes its profit function independently (taking the location of the other as given). Conversely, condition (3.2) means that if $\left(x^{*}, y^{*}\right)$ is a Nash equilibrium location-pair, then it is also an optimal location-pair.

If we can find such a pair of shadow profit functions, then the optimal location-pair of each firm will be obtained as a result of independent profit-maximization by each unit. It turns out that although it may not be easy to find a pair of shadow profit functions that always satisfy both conditions, (3.1) and (3.2), we can readily find a pair of profit functions that always satisfy condition (3.1). For example, let us define

$$
\begin{aligned}
& \Pi_{f}(x \mid y)=A(x)-R(x) S_{f}-W(x) L_{f}-\Gamma(x, y)-P(y), \\
& \Pi_{b}(y \mid x)=P(x)-R(y) S_{b}-W(y) L_{b}-\Gamma(x, y),
\end{aligned}
$$

where $P(\cdot)$ can be any function of $X{ }^{9}$ Recalling the original profit function (2.6), it can be readily verified that if we use (3.3) and (3.4), then conditions (3.1) are always satisfied.

Therefore, in the following we adopt the shadow profit functions (3.3) and (3.4), and obtain equilibrium spatial configurations in two steps. In the first step, we obtain all Nash equilibrium configrations (of the city) in which the following Nash condition

$$
\left.\begin{array}{l}
\Pi_{f}\left(x^{*} \mid y^{*}\right)=\max _{x \in X} \Pi_{f}\left(x \mid y^{*}\right) \\
\Pi_{b}\left(y^{*} \mid x^{*}\right)=\max _{y \in X} \Pi_{b}\left(y \mid x^{*}\right)
\end{array}\right\}
$$


is satisfied by the front-unit location $x^{*}$ and back-unit location $y^{*}$ of each firm. Then, in the second step, we choose the global (i.e., real) equilibrium configurations (from all Nash equilibrium configurations) in which conditions (3.2) also are satisfied by the Nash location pair of every firm.

Note from (3.3) and (3.4) that the intrafirm communication cost, $\Gamma(x, y)$, appears in the shadow profit functions of both units. This double accounting of the intrafirm communication cost is an accounting device which is essential for conditions (3.1) to be satisfied, i.e., for the optimal location pair of each firm to be sustained as a Nash equilibrium location pair. ${ }^{10}$ To see the economic meaning of these profit functions, it would be adequate to understand that $P(x)$ and $P(y)$ represent the shadow (i.e., accounting) prices of the backunit service at the front location $x$ and at the back-unit location $y$ respectively. In this context, (3.3) implies that the shadow cost (i.e., accounting cost) of the back-unit service for the front-unit locating at $x$ equals $\Gamma(x, y)+P(y)$, i.e., the sum of [the shadow price of the back-unit service at $y$ ] and [the transport cost of that service from $y$ to $x$ ]. Similarly, (3. 4) implies that the shadow net revenue of the back-unit service (produced by the back-unit) at $y$ equals $P(x)-\Gamma(x, y)$, i.e., the shadow price of that service at the front-unit location $x$ minus the transport cost of that service. Mathematically, the choice of a shadow price function, $P(\cdot)$, is completely free, i.e., it does not affect the equilibrium configuration of the city. Hence, in each part of the analysis below, we specify this function in the most convenient manner so that the economic interpretation of equilibrium conditions become easiest.

Note also that our decentralized mechanism here is essentially different from any competitive price mechanism. From (3.3), a front-unit must take the location of the associated back-unit, $y$, as given; and $y$ indeed affects the profit of the front-unit. Similarly, from (3.4), a back-unit must take the location of the associated front-unit, $x$, as given; and $x$ indeed affects the profit of the back-unit. While in a competitive price mechanism, each agent is assumed to respond to market prices only; hence, no agent is concerned about the location of any other agent.

\section{Equilibrium conditions and further specifications}

Given the context of decentralized locational choice of front-units and back-units, here we describe equilibrium conditions of land and labor markets in the city. To do so, first we introduce the bid rent function of each agent.

From (2.2), the bid rent function of households is defined as

$$
\Psi\left(x: x_{w}, z, W\left(x_{w}\right)\right)=\frac{W\left(x_{w}\right)-T\left(x, x_{w}\right)-Z}{S_{h}} .
$$

From (3.3), the bid rent function of front-units is defined as

$$
\Phi_{f}\left(x: y, A(x), W(x), P(y), \pi_{f}\right)=\frac{A(x)-W(x) L_{f}-\Gamma(x, y)-P(y)-\pi_{f}}{S_{f}},
$$

and from (3.4), that of back-units as

$$
\Phi_{b}\left(y: x, W(y), P(x), \pi_{b}\right)=\frac{P(x)-W(y) L_{b}-\Gamma(x, y)-\pi_{b}}{S_{b}} .
$$


By definition, $\Psi\left(x: x_{w}, z, W\left(x_{w}\right)\right)$ represents the maximum rent (per unit of land) which a household can bid at each residential location $x \in X$ (given its working location at $x_{w}$ ) while enjoying a consumption level, $Z$, of composite goods; here, the household also takes the wage rate $W\left(x_{w}\right)$ as given. Next, $\Phi_{f}\left(x: y, A(x), W(x), P(y), \pi_{f}\right)$ represents the maximum rent which a front-unit can bid at each location $x \in X$ (given the location of the associated back-unit at $y$ ) while enjoying a given profit level, $\pi_{f}$; here, the front-unit also takes $A(x)$, $W(x)$, and $P(y)$ as given. Similarly, $\Phi_{b}\left(y: x, W(y), P(x), \pi_{b}\right)$ represents the maximum rent which a back-unit can bid at each location $y \in X$ (given the location of the associated front-unit at $x$ ) while enjoying a given profit level, $\pi_{b}$; here, the back-unit also takes $W(y)$ and $P(x)$ as given.

Next, we enumerate unknown variables and functions. As defined before, $h(x), f(x)$, and $b(x)$ represent respectively the household density, front-unit density and back-unit density at each location $x \in X$. Similarly, $R(x)$ and $W(x)$ represent respectively the land rent and wage rate at each $x \in X$. Further, $P(x)$ represents the shadow price of the backunit service at each $x \in X{ }^{11}$

Given density functions $h(x), f(x)$, and $b(x)$ above, let their positive supports be defined respectively as

$$
h_{+} \equiv\{x \in X: h(x)>0\}, f_{+} \equiv\{x \in X: f(x)>0\}, b_{+} \equiv\{x \in X: b(x)>0\} .
$$

Then, as another set of unknown functions, we introduce firm-location correspondences, $x_{f}$ : $b_{+} \rightarrow f_{+}$, and $y_{b}: f_{+} \rightarrow b_{+}$. Here, $x=x_{f}(y)$ means that those firms with their back-units at location $y$ have their front-units at $x$; and $y=y_{b}(x)$ means that those firms with their frontunits at location $x$ have their back-units at $y$. It is always assumed that both $x_{f}$ and $y_{b}$ are onto-functions (i.e., $x_{f}\left(b_{+}\right)=f_{+}$and $y_{b}\left(f_{+}\right)=b_{+}$), and that $x_{f}$ and $y_{b}$ are inverse to each other almost everywhere (i.e., $x_{f}\left(y_{b}(x)\right)=x$ for almost all $\left.x \in f_{+}\right)$. We also introduce a commuting correspondence, $C: h_{+} \rightarrow f_{+} \cup b_{+}$; here, $x_{w}=C(x)$ means that households at $x$ commute to firms at $x_{w} . C$ is also assumed to be an onto-function (i.e., $\left.C\left(h_{+}\right)=f_{+} \cup b_{+}\right)$.

As the last set of unknowns, let $\pi_{f}(y)$ represent the (shadow) profit of a front-unit whose associated back-unit is located at $y \in X$, and $\pi_{b}(x)$ the (shadow) profit of a back-unit whose associated front-unit is located at $x \in X .^{12}$ Finally, let $Z$ be the consumption level of composite goods per household, which must be the same for all households in equilibrium.

Now, let a system,

$$
\begin{aligned}
& \left(h^{*}(x), f^{*}(x), b^{*}(x), R^{*}(x), W^{*}(x), P^{*}(x), C^{*}(x), x_{f}^{*}(y), y_{b}^{*}(x),\right. \\
& \left.\pi_{f}^{*}(y), \pi_{b}^{*}(x), Z^{*}: x, y \in X\right),
\end{aligned}
$$

represent an urban configuration (where $x_{f}^{*}$ and $y_{b}^{*}$ are inverse to each other almost everywhere). Given this configuration, for each $x, y$, and $x_{w} \in X$ let us define

$$
\begin{aligned}
& A^{*}(x) \equiv \int_{X} a[c(x, z)] f^{*}(z) d z, \\
& \Psi^{*}\left(x \mid x_{w}\right) \equiv \Psi\left(x: x_{w}, Z^{*}, W^{*}\left(x_{w}\right)\right), \\
& \Phi_{f}^{*}(x \mid y) \equiv \Phi_{f}\left(x: y, A^{*}(x), W^{*}(x), P^{*}(y), \pi_{f}^{*}(y)\right),
\end{aligned}
$$




$$
\begin{aligned}
& \Phi_{b}^{*}(y \mid x) \equiv \Phi_{b}\left(y: x, W^{*}(y), P^{*}(x), \pi_{b}^{*}(x)\right), \\
& \Pi^{*}(x, y) \equiv A^{*}(x)-R^{*}(x) S_{f}-W^{*}(x) L_{f}-R^{*}(y) S_{b}-W^{*}(y) L_{b}-\Gamma(x, y),
\end{aligned}
$$

and further define

$$
\begin{array}{ll}
h_{+}^{*} \equiv\left\{x \in X: h^{*}(x)>0\right\}, & \\
f_{+}^{*} \equiv\left\{x \in X: f^{*}(x)>0\right\}, & \\
b_{+}^{*} \equiv\left\{x \in X: b^{*}(x)>0\right\}, & \\
\Psi^{*}(x) \equiv \max _{x_{w} \in X} \Psi^{*}\left(x \mid x_{w}\right) & \text { for } x \in X, \\
\Phi_{f}^{*}(x) \equiv \max _{y \in b \neq} \Phi_{f}^{*}(x \mid y) & \text { for } x \in X, \\
\Phi_{b}^{*}(y) \equiv \max _{x \in f *} \Phi_{b}^{*}(y \mid x) & \text { for } y \in X .
\end{array}
$$

Then, we say that (4.5) represents a Nash equilibrium configuration (of the city) if the following set of conditions are satisfied:

(i) for land market equilibrium: at each $x$ and $y \in X$,

$$
\begin{aligned}
& R^{*}(x)=\max \left\{\Psi^{*}(x), \Phi_{f}^{*}(x), \Phi_{b}^{*}(x), R_{A}\right\}, \\
& h^{*}(x)>0 \Rightarrow \Psi^{*}\left(x \mid C^{*}(x)\right)=R^{*}(x), \\
& f^{*}(x)>0 \Rightarrow \Phi_{f}^{*}\left(x \mid y_{b}^{*}(x)\right)=R^{*}(x), \\
& b^{*}(x)>0 \Rightarrow \Phi_{b}^{*}\left(x \mid x_{f}^{*}(x)\right)=R^{*}(x), \\
& h^{*}(x) S_{h}+f^{*}(x) S_{f}+b^{*}(x) S_{b} \leq 1, \\
& R^{*}(x)>R_{A} \Rightarrow h^{*}(x) S_{h}+f^{*}(x) S_{f}+b^{*}(x) S_{b}=1,
\end{aligned}
$$

(ii) for labor market equilibrium:

$$
\begin{aligned}
& C^{*}\left(h_{+}^{*}\right)=f_{+}^{*} \cup b_{+}^{*}, \\
& \int_{B} h_{+}^{*}(x) d x=\int_{c(B)}^{*}\left(f^{*}(x) L_{f}+b^{*}(x) L_{b}\right) d x \\
& \quad \text { for every measurable subset } B \text { of } h_{+}^{*},
\end{aligned}
$$

(iii) for equilibrium of firm-location correspondences:

$$
\begin{aligned}
& \left.\begin{array}{l}
x_{f}^{*}\left(b_{+}^{*}\right)=f_{+}^{*}, y_{b}^{*}\left(f_{+}^{*}\right)=b_{+}^{*}, \text { and } x_{f}^{*}\left(y_{b}^{*}(x)\right)=x \\
\text { for almost all } x \in f_{+}^{*},
\end{array}\right\} \\
& \int_{B} f^{*}(x) d z=\int_{x_{*}^{*}(B)} b^{*}(x) d x \quad \text { for every measurable subset } B \text { of } f_{+}^{*},
\end{aligned}
$$


(iv) for population equilibrium:

$$
\begin{aligned}
& \int_{X} h^{*}(x) d x=N, \\
& \int_{X} f^{*}(x) d x=M=\int_{X} b^{*}(x) d x, \\
& \text { where } N=\left(L_{f}+L_{b}\right) M .
\end{aligned}
$$

Next, assuming that (4.5) is a Nash equilibrium configuration, and that it satisfies the following additional condition: there exists a constant $\pi^{*}$ such that for each $x \in X$,

$$
f^{*}(x)>0 \Rightarrow \Pi^{*}\left(x, y_{b}^{*}(x)\right)=\pi^{*}=\max _{z \in X, y \in X} \Pi^{*}(z, y) .
$$

Then, we say that (4.5) represents a (global) equilibrium configuration.

To explain the meaning of each equilibrium condition above, first let us notice that by (4.14), $\Psi^{*}(x)$ represents the maximum value of household bid rent at $x$, which is attained by optimally choosing job location $x_{w}$. Next, by the definition of $b_{+}^{*}$, every one of $M$ firms has its back-unit at some location in $b_{+}^{*}$. Therefore, in (4.15), $\Phi_{f}^{*}(x)$ represents the maximum of all front-unit bid rents at $x$ which can be paid by front-units of $M$ firms; here, by definition (4.8), a front-unit whose associated back-unit is located at $y$ must retain the given shadow profit $\pi_{f}^{*}(y)$. Similarly, every one of $M$ firms has its front-unit at some location $z \in f_{+}^{*}$. Hence, in (4.16), $\Phi_{b}^{*}(x)$ represents the maximum of all back-unit bid rents at $x$ which can be paid by back-units of $M$ firms; here, a back-unit whose associated front -unit is located at $z$ must retain the given shadow profit $\pi_{b}^{*}(z)$.

Therefore, condition (4.17) means that at each location $x$, the market rent $R^{*}(x)$ equals the maximum of the highest bid rents by four types of agents (i.e., households, front-units, back-units, and agriculture). Condition (4.18) means that if some households reside at location $x$, then their bid rent must equal the market rent; here, their job location is specified by $C^{*}(x)$. Conditions (4.19) and (4.20) have a similar meaning. Condition (4.21) represents an obvious physical land-constraint. Condition (4.22) means that if the market rent exceeds the agricultural rent, then the land must be occupied by either households or firms. Together, (4.17) $-(4.22)$ ensure that each location is occupied by the highest bidder.

Condition (4.23) means that $C^{*}(x)>0 \Leftrightarrow f^{*}\left(C^{*}(x)\right)+b^{*}\left(C^{*}(x)\right)>0$, i.e., the commuting correspondence $C^{*}$ indeed assigns a job location to each residential location. Condition (4. 24) assures that labor supply equals labor demand at each location. Next, (4.25) means that the firm-location correspondence $x_{f}^{*}$ (and $y_{b}^{*}$ ) indeed assigns a front-unit location to a back-unit location (and vice versa). Condition (4.26) assures that the firm-location correspondence $x_{f}^{*}$ always assigns the same number of back-units to any given number of frontunits. Finally, (4.27) and (4.28) represent obvious population constraints.

Let us examine important implications of conditions (4.17) to (4.20). First, it can readily be verified that together (4.17) and (4.18) imply that

$$
\begin{aligned}
& h^{*}(x)>0 \\
& \Rightarrow Z^{*}=W\left(C^{*}(x)\right)-T\left(x, C^{*}(x)\right)-R^{*} S_{b}=\max _{x w \in X}\left(W\left(w_{w}\right)-T\left(x, x_{w}\right)-R^{*}(x) S_{b}\right) .
\end{aligned}
$$

That is, the commuter correspondence $C^{*}$ assigns an optimal working location to each household in the city so that all households attain the same maximum consumption, $Z^{*}$, of 
the composite goods. Next, to see the additional implications of conditions (4.19) and (4. 20), in the same manner as (3.3) and (3.4) let us define for each $x, y \in X$,

$$
\begin{aligned}
& \Pi_{f}^{*}(x \mid y) \equiv A^{*}(x)-R^{*}(x) S_{f}-W^{*}(x) L_{f}-\Gamma(x, y)-P^{*}(y), \\
& \Pi_{b}^{*}(y \mid x) \equiv P^{*}(x)-R^{*}(y) S_{b}-W^{*}(x) L_{b}-\Gamma(x, y) .
\end{aligned}
$$

Then, we can readily see that together (4.17) and (4.19) imply that

$$
f^{*}(x)>0 \Rightarrow \pi_{f}^{*}\left(y_{b}^{*}(x)\right)=\Pi_{b}^{*}\left(x \mid y_{b}^{*}(x)\right)=\max _{z \in X} \Pi_{f}^{*}\left(z \mid y_{b}^{*}(x)\right),
$$

which means that given the back-unit location $y_{b}^{*}(x), x$ is indeed the optimal location of the associated front-unit; and $\pi_{f}^{*}\left(y_{b}^{*}(x)\right)$ equals the maximum possible profit of that front-unit. Similarly, by (4.17) and (4.20), it follows that

$$
b^{*}(y)>0 \Rightarrow \pi_{b}^{*}\left(x_{f}^{*}(y)\right)=\Pi_{b}^{*}\left(y \mid x_{f}^{*}(y)\right)=\max _{z \in X} \Pi_{b}^{*}\left(z \mid x_{f}^{*}(y)\right),
$$

which implies that given the front-unit location $x_{f}^{*}(y), y$ is indeed an optimal location of the associated back-unit; and $\pi_{b}^{*}\left(x_{f}^{*}(y)\right)$ equals the maximum possible profit of that backunit. Since $y=y_{b}^{*}(x)$ means $x=x_{f}^{*}(y)$, we can conclude from (4.25), (4.33), and (4.34) that for each $x \in f_{+}^{*},\left(x, y_{b}^{*}(x)\right)$ forms a Nash equilibrium location pair; i.e., for each $y \in b_{+}^{*},\left(x_{f}^{*}(y)\right.$, $y$ ) forms a Nash equilibrium location pair. That is, the firm-location correspondence $y_{0}^{*}$ (or $x_{f}^{*}$ ) assigns a Nash equilibrium location pair to each firm, comprised of a front-unit and back-unit.

Finally, in order to assure that the Nash equilibrium location pair of each firm is indeed a (global) optimal location pair, we must confirm that condition (4.29) is indeed satisfied for all $x \in f_{+}^{*}$. [Here, notice by (4.25) that $f^{*}(x)>0 \Leftrightarrow b^{*}\left(y_{b}(x)\right)>0$.] In (4.29), $\pi^{*}$ represents the maximum profit which can be attained by a firm choosing optimally the location of its front-unit and back-unit simultaneously. By definition of functions $\Pi^{*}, I_{f}^{*}$, and $\Pi_{b}^{*}$, the following relationship holds for all $x, y \in X$ :

$$
\Pi^{*}(x, y)=\Pi_{f}^{*}(x \mid y)+\Pi_{b}^{*}(y \mid x)+P^{*}(x)-P^{*}(y)+\Gamma(x, y) .
$$

Hence, by (4.29), (4.33), and (4.34), the following relation holds for each $x \in f_{+}^{*}$ :

$$
\pi^{*}=\pi_{f}^{*}\left(y_{b}^{*}(x)\right)+\pi_{b}^{*}(x)+P^{*}\left(y_{b}^{*}(x)\right)-P^{*}(x)+\Gamma\left(x, y_{b}^{*}(x)\right) .
$$

Therefore, if we choose an appropriate shadow price function $P^{*}$ that satisfies the following condition for each $x \in f_{+}^{*}$,

$$
P^{*}(x)=P^{*}\left(y_{b}^{*}(x)\right)+\Gamma\left(x, y_{b}^{*}(x)\right),
$$

then it follows from (4.36) that

$$
\pi^{*}=\pi_{f}^{*}\left(y_{b}^{*}(x)\right)+\pi_{b}^{*}(x) .
$$

That is, the total profit of each firm equals the sum of the shadow profits of two-units. ${ }^{13}$

In the subsequent sections, we intend to obtain explicit solutions to the equilibrium problem described. To do so, we consider a spatial case with the following simplifying assumptions on parameters: 


$$
\begin{aligned}
& X=\Re \text {, } \\
& T\left(x, x_{w}\right)=t\left|x-x_{w}\right| \quad \text { for all } x, x_{w} \in X \text {, } \\
& \Gamma(x, y)=\tau|x-y|+\delta(x, y) K \quad \text { for all } x, y \in X \text {, } \\
& \text { where } \delta(x, y)= \begin{cases}0 & \text { if } x=y \\
1 & \text { if } x \neq y\end{cases} \\
& a[c(x, y)]=\beta-\alpha|x-y| \quad \text { for all } x, y \in X \text {, }
\end{aligned}
$$

where each $t, \tau, \beta$, and $\alpha$ is a positive number, and $K$ is a non-negative number. Equation (4.38) represents a linear location space (i.e., a long-narrow city), and (4.39) means that commuting cost is a linear function of distance. In (4.40), $K$ represents the fixed cost of intrafirm communication, which becomes necessary if the two units of a firm is separated over any positive distance. Equation (4.41) represents a linear accessibility measure. ${ }^{14}$

\section{Equilibrium configurations}

In this section, we assume that in the intrafirm communication cost function (4.40), we have

$$
K=0 .
$$

That is, there exists no fixed cost of intrafirm communications. Then, each (4.39) through (4.41) represents a linear function of distance. Hence, we call this case the linear model.

For this model, it turns out that given the same set of parameters, there is a possibility that a continuum of Nash equilibrium configurations exist. ${ }^{15}$ This nonuniqueness of Nash equilibrium solution is theoretically interesting. However, since the major concern of this paper is about global equilibrium solutions, here we discuss global equilibrium configurations only. ${ }^{16}$ Hence, in the rest of this section, an equilibrium configuration means always a global one. In this context, we show the following results. First, given any set of parameters, there exists always one and only one (global) equilibrium configuration of the city. Second, in total, there exists eleven different equilibrium configurations (which are depicted in Figure 4-A to $4-\mathrm{K}$ below). Third, every equilibrium configuration can be sustained by a competitive price system in which every agent (i.e., a household, front-unit, or back-unit) follows price-signals only (without considering the location of any other agent).

Several notes are in order. It turns out that either $Z^{*}$ (equilibrium utility) or $\pi^{*}$ (equilibrium profit) cannot be determined uniquely (i.e., we can choose freely from one of them). ${ }^{17}$ Hence, in the subsequent analysis, we arbitrarily choose $\bar{Z}$ and set ${ }^{18}$

$$
Z^{*}=\bar{Z}
$$


Next, since the actual calculation for determination of each equilibrium configuration is tedious (and similar for many configurations), we demonstrate it for two configurations only in subsection 5.1, (see Ota and Fujita (1990) for calculations for the rest of configurations). In the subsequent analysis, we use the following definitions,

$$
S \equiv S_{f}+S_{b}, \quad L \equiv L_{f}+L_{b},
$$

and in figures the following notations:

$$
\begin{aligned}
& F=a \text { front-unit area, } \\
& B=a \text { back-unit area, } \\
& H=a \text { residential area. }
\end{aligned}
$$

Each $\mathrm{F}, \mathrm{B}, \mathrm{H}$ is a part (or the whole) of $f^{+}, b^{+}$, and $h^{+}$respectively.

\subsection{Examples of calculations}

We consider two land use patterns ( $\mathrm{C}$ and $\mathrm{G}$ in Figure 2. and 3 . below) and determine under what conditions (on parameters) each represents an equilibrium configuration.

\section{Pattern C}

Consider a symmetric land use pattern of which the right half is depicted in Figure 2 (a). ${ }^{19}$

(a)

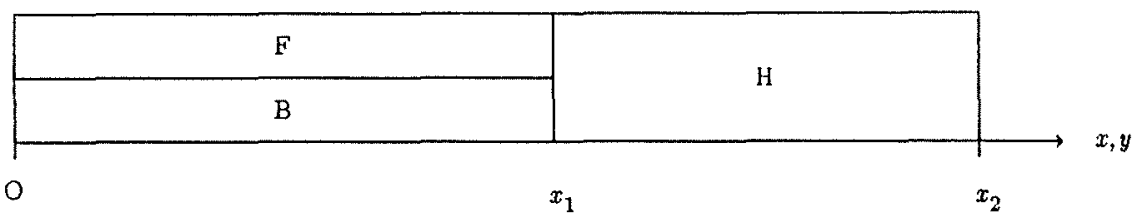

(b)

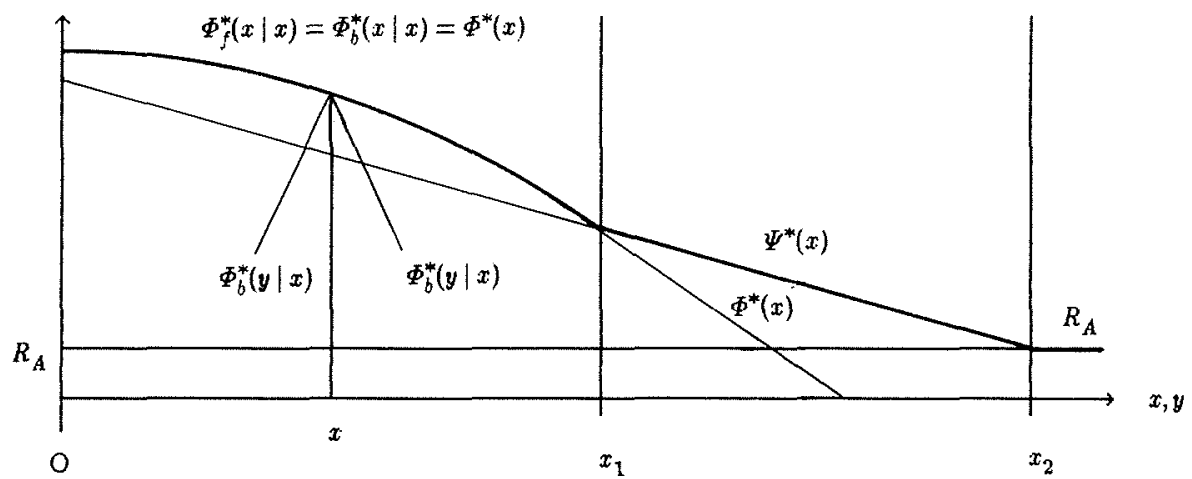

Figure 2. Equilibrium configuration of Pattern $\mathrm{C}$.

Front-units and back-units are assumed to be located together uniformly in the central area, $\left[-x_{1}, x_{1}\right]$; and households are located in the suburban areas, $\left[x_{1}, x_{2}\right]$ and $\left[-x_{2},-x_{1}\right]$. Density distributions of agents are given by 


$$
\begin{aligned}
& h^{*}(x)= \begin{cases}1 / S_{h} & \text { for } x_{1} \leq|x| \leq x_{2} \\
0 & \text { elsewhere, }\end{cases} \\
& f^{*}(x)= \begin{cases}1 / S & \text { for }|x| \leq x_{1} \\
0 & \text { elsewhere, }\end{cases} \\
& b^{*}(x)= \begin{cases}1 / S & \text { for }|x| \leq x_{1} \\
0 & \text { elsewhere }\end{cases}
\end{aligned}
$$

where, by population constraints (4.27) and (4.28), we have

$$
x_{1}=\frac{S M}{2}, \quad x_{2}=\frac{\left(S+S_{h} L\right) M}{2} .
$$

We call the land use pattern described by (5.4) to (5.7) Pattern C. Using (4.6), (5.5), and (5. 7 ), the accessibility function for this pattern can be obtained as follows:

$$
A^{*}(x)= \begin{cases}\beta M-\alpha M|x| & \text { for }|x|>x_{1} \\ \beta M-\frac{\alpha S}{4} M^{2}-\frac{\alpha}{S} x^{2} & \text { for }|x| \leq x_{1} .\end{cases}
$$

Since the front-unit and back-unit of each firm is assumed to be located together, firmlocation correspondences are given by identity-functions, i.e.

$$
x_{f}^{*}(y)=y \text { for all } y \in b_{+}^{*} \text {, and } y_{+}^{*}(x)=x \quad \text { for all } x \in f_{+}^{*} .
$$

Furthermore, given that households in area $\mathrm{H}$ commute inwardly to firms in $\mathrm{F}$ and $\mathrm{B}$ of Figure 2 (a), the commuting correspondence in given by ${ }^{20}$

$$
C^{*}(x)= \begin{cases}\left(x+x_{1}\right) x_{1} /\left(x_{2}-x_{1}\right) & \text { for }-x_{2} \leq x \leq-x_{1} \\ \left(x-x_{1}\right) x_{1} /\left(x_{2}-x_{1}\right) & \text { for } x_{1} \leq x \leq x_{2}\end{cases}
$$

It can be readily verified (Ogawa and Fujita (1980)) that in order to sustain this commuting pattern, the wage function must be such that

$$
W^{*}(x)=W^{*}-t|x|,
$$

where $W^{*}$ is an unknown constant. By (4.1), (4.7), and (5.11), the household bid rent function is given by

$$
\Psi^{*}\left(x \mid x_{w}\right)=\frac{W^{*}-t\left|x_{w}\right|-t\left|x-x_{w}\right|-\bar{Z}}{S_{h}} .
$$

Next, by appropriately choosing $P^{*}(x)$, we can always make values of $\pi_{f}^{*}(x)$ and $\pi_{b}^{*}(x)$ independent of location $x$. [In fact, such $P^{*}(x)$ is given by equation (5.23) below.] Hence, by setting $\pi_{f}^{*}(x)=\pi_{f}^{*}$ and $\pi_{b}^{*}(x)=\pi_{b}^{*}$ for each $x \in X$ (where $\pi_{f}^{*}$ and $\pi_{b}^{*}$ are still unknown), and recalling (4.2), (4.3), (4.8), and (4.9), the bid rent function of front-units and that of backunits are give respectively by

$$
\Phi_{f}^{*}(x \mid y)=\frac{A^{*}(x)-\left(W^{*}-t|x|\right) L_{f}-\tau|x-y|-P^{*}(y)-\pi_{f}^{*}}{S_{f}},
$$




$$
\Phi_{b}^{*}(y \mid x)=\frac{P^{*}(x)-\left(W^{*}-t|y|\right) L_{b}-\tau|x-y|-\pi_{b}^{*}}{S_{b}} .
$$

Equation (5.12) implies that

$$
\max _{x \in X} \Psi^{*}\left(x \mid x_{w}\right)=\frac{W^{*}-t|x|-\bar{Z}}{S_{h}} \quad \text { for each } x \in X .
$$

Furthermore, (5.10) and (5.12) together yield that

$$
\Psi^{*}\left(x \mid C^{*}(x)\right)=\frac{W^{*}-t|x|-\bar{Z}}{S_{h}} \quad \text { for each } x \in X .
$$

Therefore, recalling definition (4.14), we can conclude that

$$
\Psi^{*}(x)=\Psi^{*}\left(x \mid C^{*}(x)\right)=\frac{W^{*}-t|x|-\bar{Z}}{S_{h}} \quad \text { for each } x \in X
$$

Then, since (4.17), (4.18), and (4.22) together imply the following boundary conditions,

$$
\Psi^{*}\left(x_{2}\right)=R\left(x_{2}\right)=R_{A}
$$

we can obtain $W^{*}$ as follows:

$$
W^{*}=\bar{Z}+t x_{2}+S_{h} R_{A}
$$

This equilibrium household bid rent curve, $\Psi^{*}(x)$, is depicted in Figure 2(b).

Next, since $f^{*}(x)>0$ and $b^{*}(x)>0$ at each $x \in\left[-x_{1}, x_{1}\right]$, it must hold by (4.18), (4.19), and (5.9) that

$$
\Phi_{f}^{*}(x \mid x)=\Phi_{b}^{*}(x \mid x) \quad \text { for }|x| \leq x_{1},
$$

where

$$
\begin{aligned}
& \Phi_{f}^{*}(x \mid x)=\frac{A^{*}(x)-\left(W^{*}-t|x|\right) L_{f}-P^{*}(x)-\pi_{f}^{*}}{S_{f}}, \\
& \Phi_{b}^{*}(x \mid x)=\frac{P^{*}(x)-\left(W^{*}-t|x|\right) L_{b}-\pi_{b}^{*}}{S_{b}} .
\end{aligned}
$$

Relation (5.20) means that since both units of each firm are located together, they should have the same bid rent at each $x \in\left[-x_{1}, x_{1}\right]$. Now, solving equations (5.20) to (5.22) for $P^{*}(x)$, we can obtain

$$
P^{*}(x)=\frac{\left(A^{*}(x)-W^{*}(x) L_{f}-\pi_{f}^{*}\right) S_{b}+\left(W^{*}(x) L_{b}-\pi_{b}^{*}\right) S_{f}}{S} \text { for }|x| \leq x_{1},
$$

where $W^{*}(x)=W^{*}-t|x|$. Although the value of $P^{*}(x)$ does not matter in the area where $|x|>x_{1}$ (because no firm is located there in equilibrium), for convenience we define

$$
P^{*}(x)=P^{*}\left(x_{1}\right)-\tau|x| \quad \text { for }|x|>x_{1} .
$$

Next, in the global equilibrium condition (4.36), if we use (5.9) and set $\pi_{f}^{*}(y)=\pi_{f}^{*}$ and $\pi_{b}^{*}(x)=\pi_{b}^{*}$, then we have

$$
\pi^{*}=\pi_{f}^{*}+\pi_{b}^{*}
$$

which means that the (total) profit of each firm equals the sum of the shadow profits of the two units. Now, if we define the combined bid rent function, $\Phi^{*}(x)$, by 


$$
\Phi^{*}(x) \equiv \frac{A^{*}(x)-\left(W^{*}-t|x|\right) L-\pi^{*}}{S} \quad \text { for each } x \in X,
$$

then we can readily see that $(5.20)$ implies

$$
\Phi_{f}^{*}(x \mid x)=\Phi_{b}^{*}(x \mid x)=\Phi^{*}(x) \quad \text { for }|x| \leq x_{1} .
$$

This relation is depicted in Figure 2(b). By definition, $\Phi^{*}(x)$ represents the maximum rent (per unit of land) which a firm can pay at each $x$ when both units of the firm are located together (while achieving the equilibrium profit $\pi^{*}$ ).

Recall that the land-market equilibrium conditions (4.17) to (4.22) together mean, in short, that each location is to be occupied by agents with the highest bid rent. Using functions (5.13), (5.14), (5.17), and (5.26) above, this requirement can be restated into the following set of conditions:

(a) $\Phi^{*}(x) \geq \Psi^{*}(x)$ for all $|x| \leq x_{1}$, and $\Phi^{*}(x) \leq \Psi^{*}(x)$ for all $|x| \geq x_{1}$.

(b) Given any $x$ such that $|x| \leq x_{1}$, we have $\Phi_{b}^{*}(y \mid x) \leq \max \left\{\Phi^{*}(y), \Psi^{*}(y), R_{A}\right\}$ for all $y \in X$.

(c) Given any $x$ such that $|x| \leq x_{1}$, we have $\Phi_{f}^{*}(z \mid x) \leq \max \left\{\Phi^{*}(z), \Psi^{*}(z), R_{A}\right\}$ for all $z \in X$.

Notice from (5.17) that $\Psi^{*}(x)$ represents a straight line (on each side of 0 ), and from (5.8) and (5.26) that $\Phi^{*}(x)$ is strictly concave on $\left[-x_{1}, x_{1}\right]$ and linear in the rest of $X$. Therefore, as can be seen from Figure 2(b), condition (a) is satisfied if and only if

$$
\begin{aligned}
& \Phi^{*}\left(x_{1}\right)=\Psi^{*}\left(x_{1}\right), \text { and } \\
& \Phi^{*}(0) \geq \Psi^{*}(0) .
\end{aligned}
$$

Equation (5.28) yields that

$$
\pi^{*}=\beta M-\frac{\alpha S}{2} M^{2}-W^{*} L-S R_{A}
$$

and we can readily see that relation (5.29) holds if and only if

$$
t \leq \frac{S_{h} M}{2\left(S+S_{h} L\right)} \alpha
$$

Next, condition (b) means the following (refer to Figure 2(b)): Take any firm of which front-unit locates at some location $x$ in the area $\left[-x_{1}, x_{1}\right]$ ( $\equiv f_{+}^{*}$, which is the location area of firms). Given the front-unit location at $x$, by changing the location of the associated back-unit to various points $y$ in $X$ we can obtain the bid rent curve of this back-unit over $X$. As can be seen from (5.14), given fixed $x, \Phi_{b}^{*}(y \mid x)$ represents an inverse-V-shaped curve (which is depicted in Figure 2(b)). In this context, condition (b) means that given any $x \in$ $\left[-x_{1}, x_{1}\right]$, this back-unit bid rent curve, $\Phi_{b}^{*}(y \mid x)$, is always below the envelope of the three curves, $\Phi^{*}(x), \Psi^{*}(x)$, and $R_{A}$. By (5.14),

$$
\frac{\partial \Phi_{b}^{*}(y \mid x)}{\partial y}= \begin{cases}\left(t L_{b}-\tau\right) / S_{b} & \text { for } 0 \leq x<y \\ \left(t L_{b}+\tau\right) / S_{b} & \text { for } 0<y<x \\ \left(-t L_{b}+\tau\right) / S_{b} & \text { for } y<0 \leq x\end{cases}
$$


and by (5.26),

$$
\frac{d \Phi^{*}(x)}{d x}=\frac{-(2 \alpha x / S)+t L}{S} \text { for } x>0
$$

which is decreasing in $x$. Hence, this condition of dominance (of $\Phi_{b}^{*}(y \mid x)$ by $\max \left\{\Phi^{*}(x)\right.$, $\left.\left.\Psi^{*}(x), R_{A}\right\}\right)$ is satisfied if and only if the following set of conditions are satisfied (refer to Figure 2(b)):

$$
\begin{aligned}
& \frac{-\left(2 \alpha x_{1} / S\right)+t L}{S} \equiv \frac{d \Phi^{*}(x)}{d x} \geq \frac{\partial \Phi_{b}^{*}\left(x_{1} \mid x\right)}{\partial y_{+}} \equiv \frac{t L_{b}-\tau}{S_{b}}, \\
& \frac{t L}{S} \equiv \frac{d \Phi^{*}(0)}{d x_{+}} \leq \frac{\partial \Phi_{b}^{*}(0 \mid x)}{\partial y_{-}} \equiv \frac{t L_{b}+\tau}{S_{b}}, \\
& \Phi_{b}^{*}(y \mid x) \leq \Psi^{*}(y) \quad \text { for all } y \leq 0 \leq x .
\end{aligned}
$$

Using (5.7) and (5.34), we have

$$
\tau \geq \frac{S_{b} M}{S} \alpha-\frac{S_{b} L_{f}-S_{f} L_{b}}{S} t
$$

We can readily see that condition (5.35) is always satisfied if (5.31) and (5.37) are satisfied, and that together (5.31) and (5.37) imply

$$
\tau>\frac{S_{b}+S_{h} L_{b}}{S_{h}} t \text {. }
$$

Using (5.38), it can be readily verified that condition (5.36) is always satisfied if (5.29) is satisfied. Therefore, we can conclude that condition (b) is satisfied if and only if conditions (5.30), (5.31), and (5.37) are satisfied.

Next, condition (c) means that given a fixed location of a back-unit at any $x \in\left[-x_{1}\right.$, $\left.x_{1}\right]$, the bid rent curve, $\Phi_{f}^{*}(z \mid x)$, of the associated front-unit is always dominated by at least one of three curves, $\Phi^{*}(z), \Psi^{*}(z)$, and $R_{A}$. In a manner similar to (b) the above (i.e., by examining the sign of $\left[\partial \Phi_{f}^{*}(z \mid x) / \partial z-\partial \Phi^{*}(z) / \partial z\right]$ at each $z \in X$ for each $\left.x \in\left[-x_{1}, x_{1}\right]\right)$, it can be readily verified that if conditions (5.30), (5.31), and (5.37) are satisfied, then condition (c) is always satisfied. ${ }^{21}$ Therefore, summarizing the results, we can see that three conditions, (a), (b), and (c), can be satisfied if and only if (5.30), (5.31), and (5.37) are satisfied. Then, since (5.30) is the equation that defines the equilibrium value of $\pi^{*}$, only (5.31) and (5.37) represent the set of parameter conditions that are necessary for Pattern $C$. Therefore, we can conclude that Pattern $C$ represents an equilibrium land use configuration if and only if parameter conditions, (5.31) and (5.37), are satisfied. This parameter region for Pattern $\mathrm{C}$ is denoted by area $\mathrm{C}$ in Figure 5 below.

\section{Pattern G}

Next, consider a symmetric land use pattern of which right half is depicted in Figure 3(a). All front-units are located together in the central area, $\left[-x_{1}, x_{1}\right]$, which forms the CBD. Workers for the CBD commute from the surrounding residential areas, $\left[-x_{2},-x_{1}\right]$ and $\left[x_{1}, x_{2}\right]$. All back-units together with their workers are located in the far suburbs, $\left[-x_{3}\right.$, $\left.-x_{2}\right]$ and $\left[x_{2}, x_{3}\right]$; no commuting occurs in these areas. Density distributions of agents are given by 
(a)

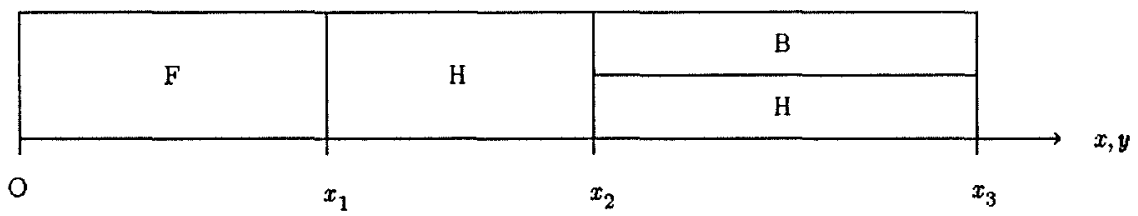

(b)

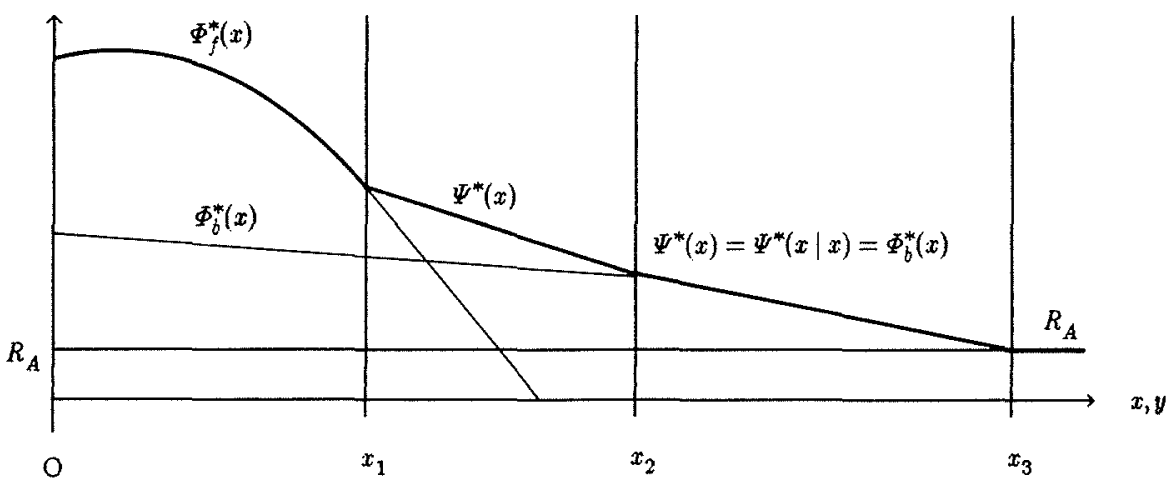

Figure 3. Equilibrium configuration of Pattern G.

$$
\begin{aligned}
& h^{*}(x)= \begin{cases}1 / S_{h} & \text { for } x_{1} \leq|x| \leq x_{2} \\
L_{b} /\left(S_{h} L_{b}+S_{b}\right) & \text { for } x_{2} \leq|x| \leq x_{3} \\
0 & \text { elsewhere, }\end{cases} \\
& f^{*}(x)= \begin{cases}1 / S_{f} & \text { for }|x| \leq x_{1} \\
0 & \text { elsewhere, }\end{cases} \\
& b^{*}(x)= \begin{cases}1 /\left(S_{h} L_{b}+S_{b}\right) & \text { for } x_{2} \leq|x| \leq x_{3} \\
0 & \text { elsewhere, }\end{cases}
\end{aligned}
$$

where, by population constraints (4.27) and (4.28), we have

$$
x_{1}=\frac{S_{f} M}{2}, x_{2}=\frac{\left(S_{f}+S_{h} L_{f}\right) M}{2}, x_{3}=\frac{\left(S+S_{h} L\right) M}{2} .
$$

The land use pattern described by (5.39) to (5.42) is called Pattern G. Using (4.6), (5.40), and (5.42), the accessibility function for this pattern can be obtained as

$$
A^{*}(x)= \begin{cases}\beta M-\alpha M|x| & \text { for }|x| \geq x_{1} \\ \beta M-\frac{\alpha S_{f}}{4} M^{2}-\frac{\alpha}{S_{f}} x^{2} & \text { for }|x| \leq x_{1} .\end{cases}
$$

Since front-units in area $\left[0, x_{1}\right]$ (in area $\left[-x_{1}, 0\right]$ ) are associated with back-units in area $\left[x_{2}\right.$, $\left.x_{3}\right]$ (in area $\left[-x_{3},-x_{2}\right]$ ), firm-location correspondences are given by 


$$
\begin{aligned}
& x_{f}^{*}(y)= \begin{cases}\left(y+x_{2}\right) x_{1} /\left(x_{3}-x_{2}\right) & \text { for }-x_{3} \leq x \leq-x_{2} \\
\left(y-x_{2}\right) x_{1} /\left(x_{3}-x_{2}\right) & \text { for } x_{2} \leq y \leq x_{3},\end{cases} \\
& y_{b}^{*}(x)= \begin{cases}\left(x_{3}-x_{2}\right) x / x_{1}-x_{2} & \text { for }-x_{1} \leq x<0 \\
\left(x_{3}-x_{2}\right) x / x_{1}+x_{2} & \text { for } 0 \leq x<x_{1} .\end{cases}
\end{aligned}
$$

The commuting correspondence is given by

$$
C^{*}(x)= \begin{cases}\left(x+x_{1}\right) x_{1} /\left(x_{2}-x_{1}\right) & \text { for }-x_{2}<x \leq-x_{1} \\ \left(x-x_{1}\right) x_{1} /\left(x_{2}-x_{1}\right) & \text { for } x_{1} \leq x<x_{2} \\ x & \text { for } x_{1} \leq|x| \leq x_{2}\end{cases}
$$

Setting $Z^{*}=\bar{Z}, \pi_{f}^{*}(y)=\pi_{f}^{*}$, and $\pi_{b}^{*}(y)=\pi_{b}^{*}$ in equations (4.7), (4.8), and (4.9), we have the following bid rent functions:

$$
\begin{aligned}
& \Psi^{*}\left(x \mid x_{w}\right)=\frac{W^{*}\left(w_{w}\right)-t\left|x-x_{w}\right|-\bar{Z}}{S_{h}}, \\
& \Phi_{f}^{*}(x \mid y)=\frac{A^{*}(x)-W^{*}(x) L_{f}-\tau|x-y|-P^{*}(y)-\pi_{f}^{*}}{S_{f}}, \\
& \Phi_{b}^{*}(y \mid x)=\frac{P^{*}(x)-W^{*}(y) L_{b}-\tau|x-y|-\pi_{b}^{*}}{S_{b}} .
\end{aligned}
$$

First, we determine the spatial structure of the shadow price function, $P^{*}(x)$. Since $f_{+}^{*}=$ $\left[-x_{1}, x_{1}\right]$, it must hold by (4.16), (4.17), and (4.20) that

$$
\Phi_{b}^{*}\left(x \mid x_{f}^{*}(x)\right)=\Phi_{b}^{*}(x) \equiv \max _{-x_{1} \leq z \leq x_{1}} \Phi_{b}^{*}(x \mid z) \quad \text { for } x_{2} \leq|x| \leq x_{3},
$$

which implies that

$$
P^{*}\left(x_{f}^{*}(x)\right)-\tau\left|x_{f}^{*}(x)-x\right|=\max _{-x_{1} \leq z \leq x_{1}}\left(P^{*}(z)-\tau|z-x|\right) \quad \text { for } x_{2} \leq|x| \leq x_{3} .
$$

Using the function $x_{f}^{*}(x)$ given by (5.44), we can readily see that for the relation (5.51) to hold for $x_{2} \leq|x| \leq x_{3}, P^{*}(x)$ must be such that

$$
P^{*}(x)=P^{*}-\tau|x| \quad \text { for }|x| \leq x_{1}
$$

where $P^{*}$ is an unknown constant. Next, together (4.15), (4.17), and (4.19) imply that

$$
\Phi_{f}^{*}\left(x \mid y_{b}^{*}(x)\right)=\Phi_{f}^{*}(x) \equiv \max _{x_{2} \leq|y| \leq x_{3}} \Phi_{f}^{*}(x \mid y) \quad \text { for }|x| \leq x_{1} .
$$

In a manner similar to the above, using (5.45) and (5.51), we can obtain the following relation:

$$
P^{*}(x)=P^{* *}-\tau|x| \quad \text { for } x_{2} \leq|x| \leq x_{3},
$$

where $P^{* *}$ is another unknown constant. It is not difficult to see from the forms of bid rent functions (5.48) and (5.49) that by appropriately choosing $\pi_{f}^{*}$ and $\pi_{b}^{*}$, we can require that $P^{*}$ $=P^{* *}$. Furthermore, the value of $P^{*}(x)$ in the area where no firm is located cannot be determined uniquely. Hence, without loss of generality, we can assume that

$$
P^{*}(x)=P^{*}-\tau|x| \quad \text { for all } x \in X
$$


Then, using (5.55) and definitions (4.15) and (4.16), we can obtain the following (maximum) bid rent curves of front-units and back-units respectively:

$$
\begin{array}{ll}
\Phi_{f}^{*}(x)=\frac{A^{*}(x)-W^{*}(x) L_{f}-\left(P^{*}-\tau|x|-\pi_{f}^{*}\right)}{S_{f}} & \text { for } x \in X, \\
\Phi_{b}^{*}(x)=\frac{P^{*}-\tau|x|-W^{*}(x) L_{b}-\tau_{b}^{*}}{S_{b}} & \text { for } x \in X .
\end{array}
$$

These curves are depicted in Figure 3(b).

Next, we define the spatial structure of the wage function, $W^{*}(x)$. Again, similar to the report by Ogawa and Fujita (1980), we can see that in order to sustain the commuting pattern given by (5.46), it must hold that

$$
W^{*}(x)=W^{*}-t|x| \quad \text { for }|x| \leq x_{2},
$$

and

$$
\left|\frac{d W^{*}(x)}{d x}\right| \leq t \quad \text { for all } x \in X
$$

Condition (5.59) implies that

$$
\Psi^{*}(x) \equiv \max _{x w \in X} \Psi^{*}\left(x \mid x_{w}\right)=\Psi^{*}(x \mid x) \quad \text { for all } x \in X .
$$

Therefore, by (5.47) and (5.58),

$$
\Psi^{*}(x)=\frac{W^{*}-t|x|-\bar{Z}}{S_{h}} \quad \text { for }|x| \leq x_{2},
$$

which gives the household bid-rent curve in the commuting area, $|x|<x_{2}$ (refer to Figure 3(b)). Next, as depicted in Figure 3(b), in the noncommuting area where $x_{2} \leq|x| \leq x_{3}$, households and back-units should have the same bid rent:

$$
\Psi^{*}(x)=\Phi_{b}^{*}(x) \quad \text { for } x_{2} \leq|x| \leq x_{3} .
$$

Hence, using (5.60), we have that $\Psi^{*}(x \mid x)=\Phi_{b}^{*}(x)$ for $x_{2}<|x|<x_{3}$, or

$$
\frac{W^{*}(x)-\bar{Z}}{S_{h}}=\frac{P^{*}-\tau|x|-W^{*}(x) L_{b}-\pi_{b}^{*}}{S_{b}} \quad \text { for } x_{2} \leq|x| \leq x_{3},
$$

which yields that

$$
W^{*}(x)=\frac{\left(P^{*}-\pi_{b}^{*}\right) S_{h}+\bar{Z} S_{b}}{S_{b}+S_{h} L_{b}}-\frac{S_{h}}{S_{b}+S_{h} L_{b}} \tau|x| \quad \text { for } x_{2} \leq|x| \leq x_{3} .
$$

Setting $x=x_{2}$, by (5.57) and (5.62), we have

$$
W^{*}=\frac{\left(P^{*}-\pi_{b}^{*}\right) S_{h}+\bar{Z} S_{b}}{S_{b}+S_{h} L_{b}}-\frac{\left(S_{f}+S_{h} L_{f}\right) M S_{h}}{2\left(S_{b}+S_{h} L_{b}\right)} \tau+\frac{\left(S_{f}+S_{h} L_{f}\right) M}{2} t .
$$

Considering (5.59), without loss of generality we can set $W^{*}(x)=W^{*}\left(x_{3}\right)-t|x|$ for $|x| \geq x_{3}$. In summary, we have

$$
W^{*}(x)= \begin{cases}W^{*}-t|x| & \text { for }|x| \leq x_{2} \\ \frac{\left(P^{*}-\pi_{b}^{*}\right) S_{h}+\bar{Z} S_{b}}{S_{b}+S_{h} L_{b}}-\frac{S_{h}}{S_{b}+S_{h} L_{b}} \tau|x| & \text { for } x_{2} \leq|x| \leq x_{3} \\ W^{*}\left(x_{3}\right)-t|x| & \text { for }|x| \geq x_{3}\end{cases}
$$

where $W^{*}$ is given by $(5.64)$. 
The value of $\left(P^{*}-\pi_{b}^{*}\right)$ in (5.64) and (5.65) can be determined as follows. By the boundary rent condition, $\Psi^{*}\left(x_{3} \mid x_{3}\right)=R_{A}$, we have that $W^{*}\left(x_{3}\right)=\bar{Z}+R_{A} S_{h}$. Hence, by (5.65), we have

$$
P^{*}-\pi_{b}^{*}=R_{A} S_{b}+\left(\bar{Z}+R_{A} S_{h}\right) L_{b}+\frac{S+S_{h} L}{2} \tau M .
$$

Finally, since the two bid rent curves, $\Phi_{f}^{*}(x)$ and $\Psi: *(x)$, must meet at $x=x_{1}$ (refer to Figure 3(b)), by setting $\Phi_{f}^{*}\left(x_{1}\right)=\Psi^{*}\left(x_{1}\right)$, we can obtain

$$
P^{*}+\pi_{f}^{*}=\beta M-\frac{\alpha S_{f}}{2} M^{2}-W^{*} L_{f}-S_{f} R_{A},
$$

and hence

$$
\begin{aligned}
\pi^{*}= & \pi_{f}^{*}+\pi_{b}^{*}=\beta M-\frac{\alpha S_{f}}{2} M^{2} \\
& -\left(W^{*} L_{f}+\frac{\left(P^{*}-\pi_{b}^{*}\right) S_{h}+\bar{Z} S_{b}}{S_{b}+S_{h} L_{b}} L_{b}\right)-S R_{A}-\frac{S_{b}\left(S+S_{h} L\right) M}{2\left(S_{b}+S_{h} L_{b}\right)} \tau .
\end{aligned}
$$

Now, recalling condition (5.59) and referring to Figure $3(\mathrm{~b})$, we can readily see that for Pattern $\mathrm{G}$ to represent an equilibrium configuration, it is necessary and sufficient that the following three conditions be satisfied:
(a) $\left|\frac{d W^{*}(x)}{d x}\right| \leq t$,
(b) $\Phi_{f}^{*}(0) \geq \Psi^{*}(0)$,
(c) $\Psi^{*}\left(x_{1}\right) \geq \Phi_{b}^{*}\left(x_{1}\right)$.

By (5.65), condition (a) means that $S_{h} \tau /\left(S_{b}+S_{h} L_{b}\right) \leq t$ or

$$
\tau \leq \frac{S_{b}+S_{h} L_{b}}{S_{h}} t
$$

Condition (b) leads to

$$
\tau \leq \frac{\alpha M}{2}-\frac{S_{b}+S_{h} L_{b}}{S_{h}} t
$$

It is not difficult to see that condition (c) is satisfied if and only if (5.69) holds.

Therefore, we can conclude that Pattern $G$ represents an equilibrium land use configuration if and only if parameter conditions, (5.69) and (5.70), are satisfied. This parameter region for Pattern $G$ is denoted by area $G$ in Figure 5 below.

If we assume that all back-units supply a homogeneous service, called the back-unit service, to front-units, then both equilibrium configurations of Patterns $C$ and $G$ can be supported by competitive price systems. To see this, assuming that [instead of shadow profit functions (3.3) and (3.4)], the (real) profit function of each front-unit is given by

$$
\tilde{\pi}_{f}=A(x)-R(x) S_{f}-W(x) S_{f}-P(x)
$$

and that of each back-unit by

$$
\tilde{\pi}_{b}(y, x)=P(x)-R(y) S_{b}-W(y) L_{b}-\Gamma(x, y) .
$$

In contrast to (3.3), we assume by the profit function (5.71) that each front-unit purchases the back-unit service at its location, $x$, at a given market price, $P(x)$. By (5.72), we assume 
that each back-unit (locating at $y$ ) is free to choose a location, $x$, for sale of its back-unit service. It is not difficult to see that if we use $P^{*}(x)$ given by (5.23) and (5.24) as the market price of back-unit service at each location, then the land use configuration of Pattern $\mathrm{C}$ can be attained as a competitive equilibrium (provided parameter conditions (5.31) and (5.37) are satisfied). Similarly, under the back-unit service price $P^{*}(x)$ given by (5.55), the land use configuration of Pattern $G$ can be attained as a competitive equilibrium. More generally, it can be verified that any global equilibrium configuration can be attained as a competitive equilibrium. ${ }^{23}$

\subsection{Comparison of equilibrium configurations}

Figure 4-A through 4-K depict all possible equilibrium configurations. The upper part of each figure depicts a land use configuration, and the lower part the associated land rent configuration. The parameter region of each equilibrium configuration is denoted in Figure 5.

Pattern A represents a completely mixed configuration, in which all three types of agents (i.e., front-units, back-units, households) are located in the same proportion everywhere in the city, that is, each front-unit is located together with its back-unit and its workers.

In Patterns B to F, the city is surrounded by pure residential areas on both sides of the furthest suburbs. Front-units and back-units are located always closely. As we can see from Figure 5, these five patterns occur when $\tau>\left(\left(S_{b}+S_{h} L_{b}\right) / S_{h}\right) t$, that is, when the intrafirm commuting cost rate, $\tau$, is relatively high compared to the commuting cost rate, $t$.

In Patterns $G$ to $K$, both sides of the furthest suburbs consist of back-unit area in which back-units are located together with their workers. As can be seen from Figure 5, these five patterns occur when $\tau>\left(\left(S_{b}+S_{h} L_{b}\right) / S_{h}\right) t$, i.e., when the intrafirm commuting cost rate is relatively low compared to the commuting cost rate.

In Figure 5, as we move towards the upper-right direction, i.e., as both the commuting cost rate $t$ and intrafirm communication cost rate $\tau$ increase simultaneously, which is unexpected, more mixture of three activities occurs.

We can also observe the following results from Figure 5:

(i) Given any value of $M \alpha$ (= the firm-population size multiplied by the interfirm communication cost rate), the $(t, \tau)$-parameter space is always divided into the same number (eleven) of regions (i.e., equilibrium configurations.)

(ii) Furthermore, the parameter region of each equilibrium configuration expands towards the upper-right direction proportionally to $M \alpha$.

(iii) In turn, this implies that given any fixed combination of $(t, \tau)$, as $M \alpha$ continues to increase (e.g., the firm-population size $M$ increases), the equilibrium configuration of the city becomes eventually either Pattern $F$ or $G$.

(iv) Given any fixed combination of $t$ and $M \alpha$, as $\tau$ keeps decreasing (i.e., as the intrafirm communication technology continues to improve), the equilibrium configuration of the city becomes eventually either Pattern G, H, or I. In either one of these three patterns, the far suburbs are occupied by back-units and their workers. Therefore, the advancement of 

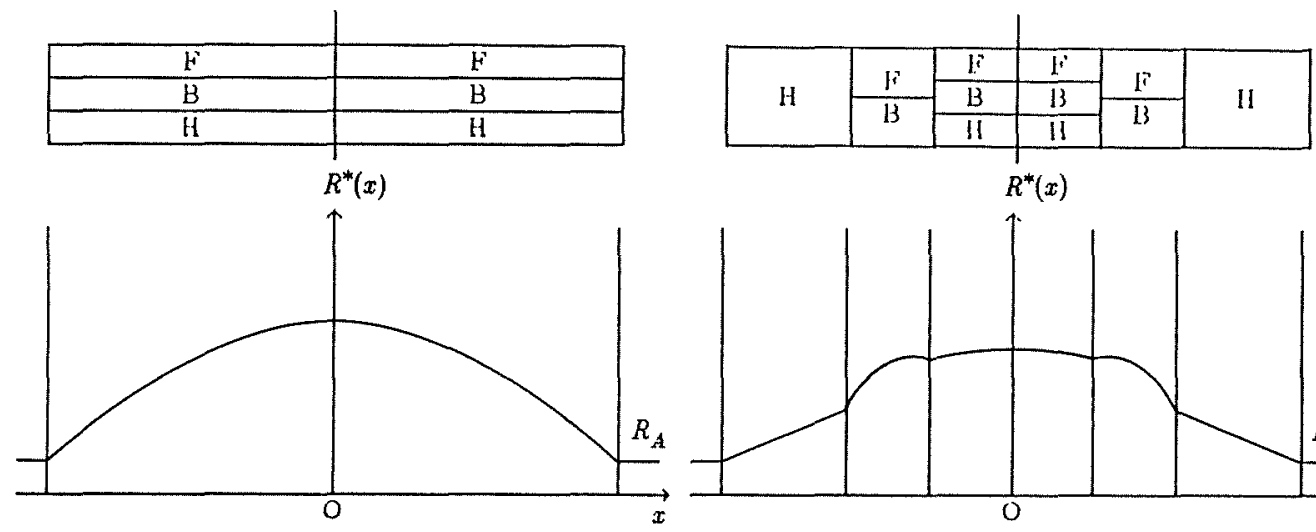

Figure 4-A : Pattern A.

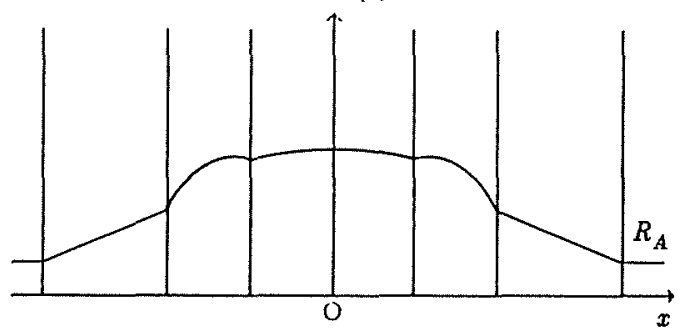

Figure 4-B : Pattern B.
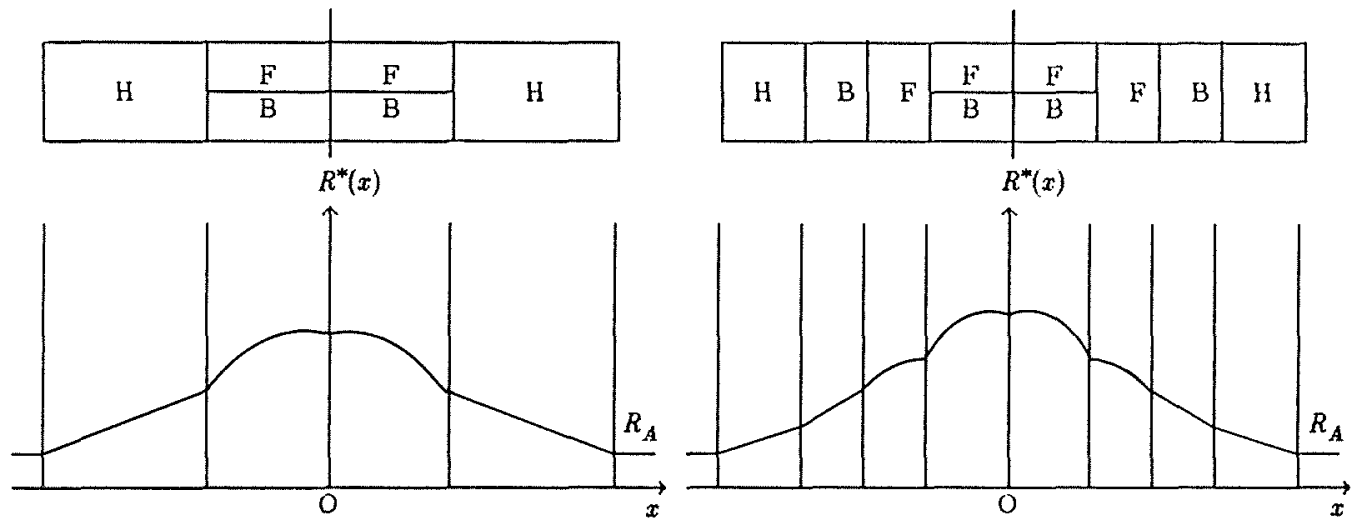

Figure 4-C : Pattern C.

Figure 4-D : Pattern D.
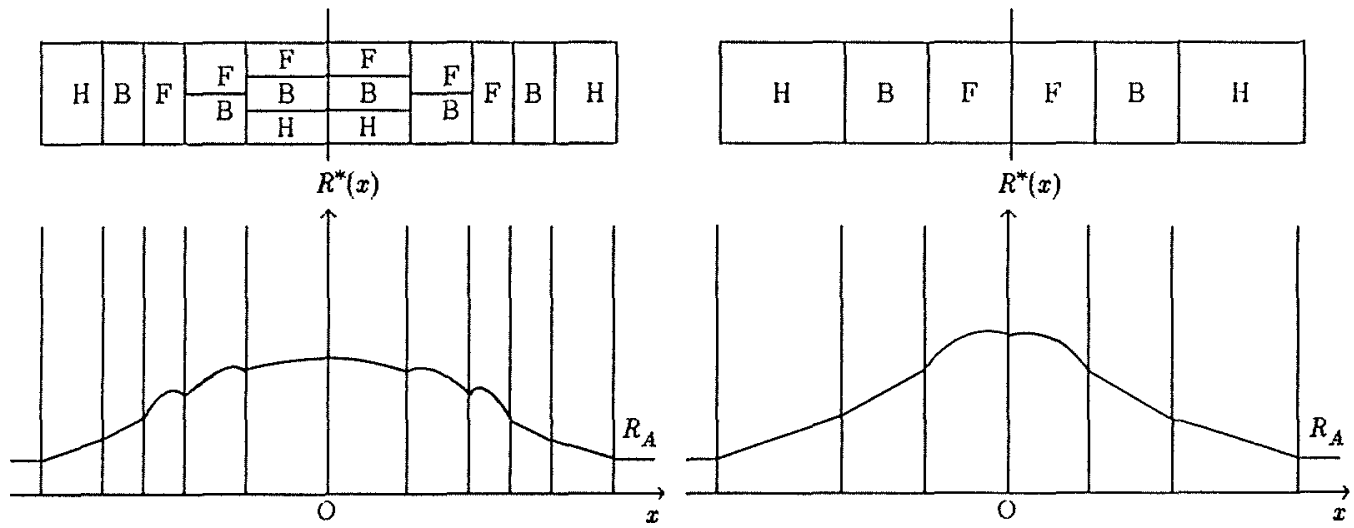

Figure 4-E : Pattern E.

Figure 4-F : Pattern F. 

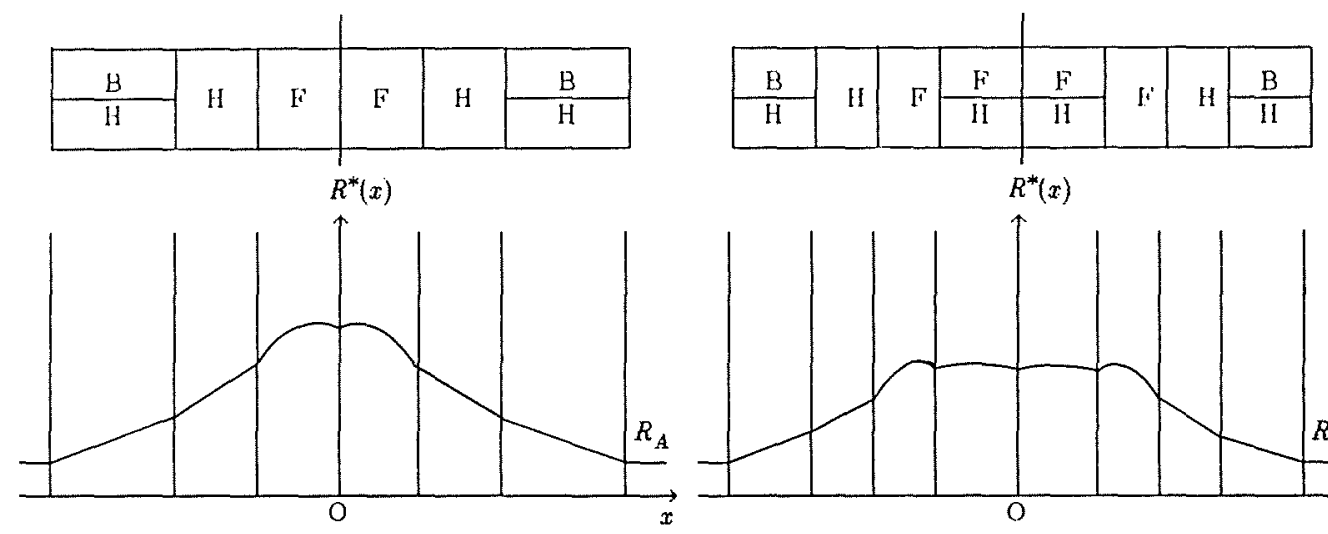

Figure 4-G : Pattern $G$.



Figure 4-H : Pattern H
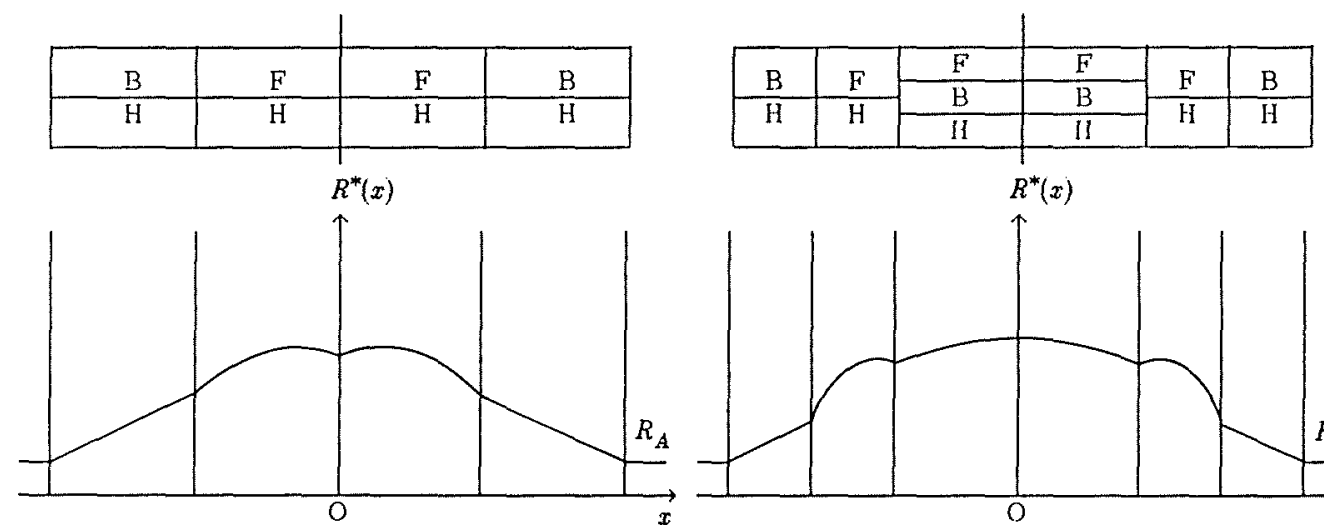

Figure 4-I : Pattern I.

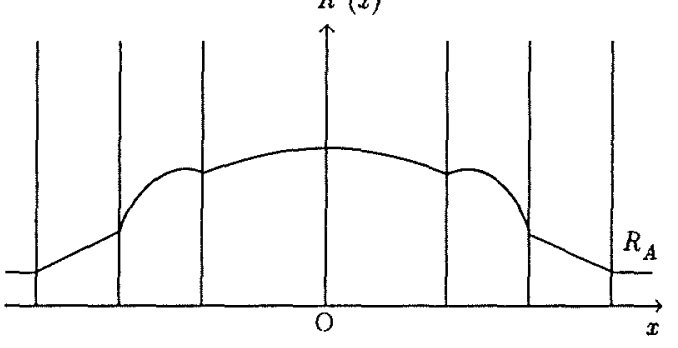

Figure 4-J : Pattern J.
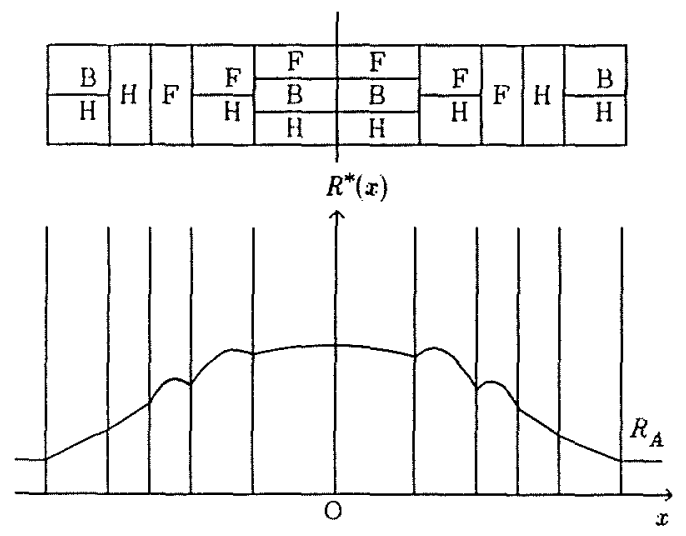

Figure 4-K : Pattern $\mathrm{K}$. 


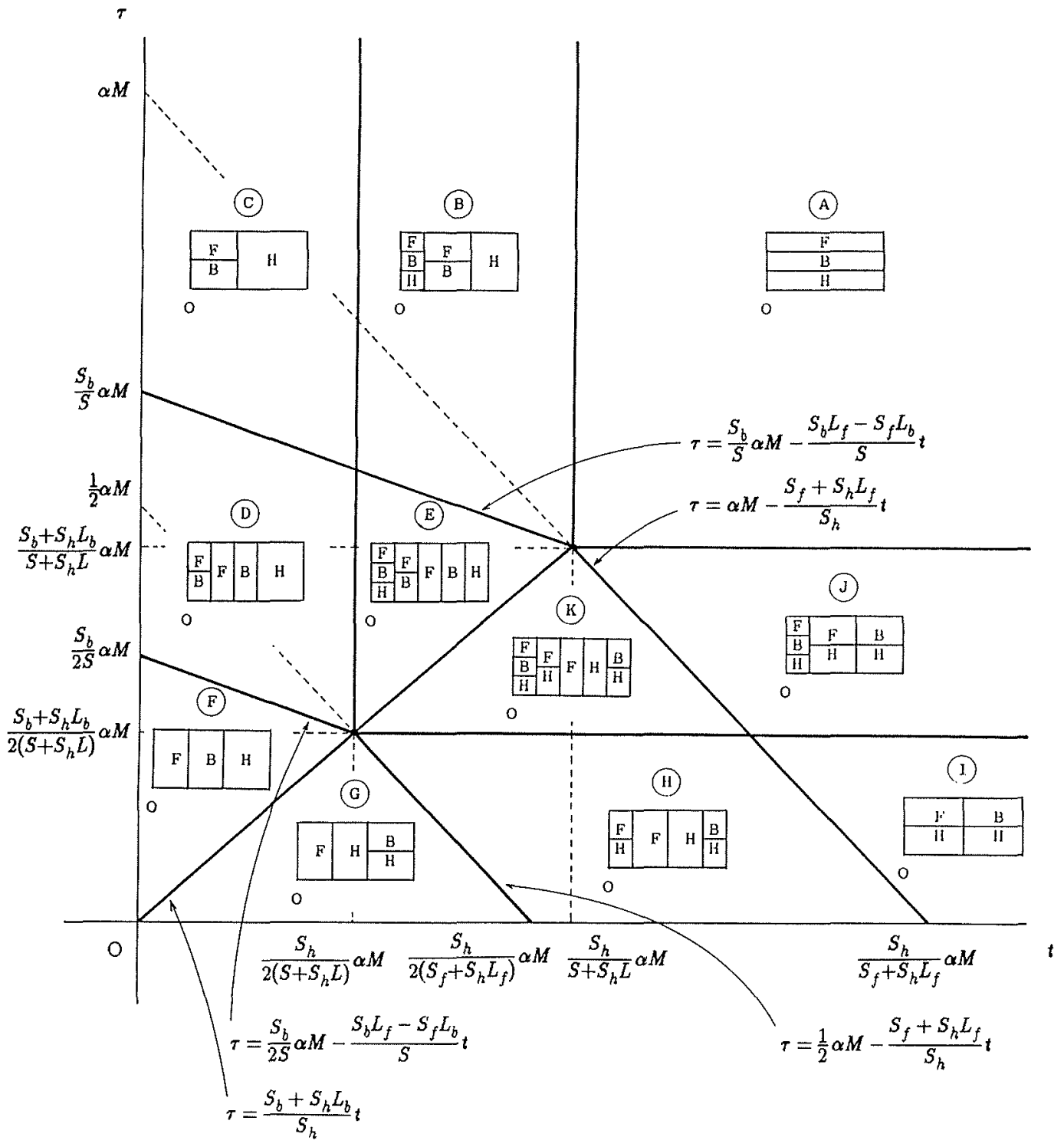

Figure 5. Parameter regions for equilibrium configurations,

intrafirm communication technologies provides a major cause of job-suburbanization.

\section{Conclusion}

In this paper, we have developed a general equilibrium model of the city, in which each firm consists of a front-unit and back-unit. We have shown that depending on the parameters, eleven different equilibrium configurations of the city emerge. In particular, it has been demonstrated that the advancement of intrafirm communication technology 
will eventually lead to dichotomy of firm activities, where the front-unit activity (specialized in extrafirm communications) will concentrate in the city center, while the back-unit activity will be located in the far suburbs.

Our model presented in this paper is relatively simple, and it is desirable to extend the model into the following directions. First, although we have assumed that there exists no fixed cost of intrafirm communications, (i.e., $K=0$ in (4.40)) this assumption is not very realistic, that is, intrafirm communications between a front-unit and back-unit may be hampered significantly even if the two units are located a block away. Hence, it is important to introduce the fixed-cost of intrafirm communications. With $K>0$, some of previous eleven configurations will not represent equilibrium solutions any more. Second, you will note that in any one of eleven equilibrium configurations, all front-units are located contiguously (i.e., in each Figure $4-\mathrm{A}$ to $4-\mathrm{K}$, the $\mathrm{F}$-area is always connected). That is, in the context of the present paper, no subcenter emerges in the city. This result is due to the assumption of a linear accessibility measure (represented by (4.42)). With the introduction of a nonlinear accessibility measure, the formation of subcenters may become possible. For an extension in this direction, the work by Fujita and Ogawa (1982) will be useful. Third, we need to replace fixed-coefficient functions with more general utility and production functions. Finally, in this paper we have considered one type of labor and one type of firm only. With the introduction of multiple types of labor and firm, our model will become more useful for the explanation of actual spatial configurations of metropolitan areas.

\section{Footnotes}

1. We hasten to note that there are several papers on spatial pricing and spatial competition which consider location of multi-outlet firms (for a survey, see, for example, Gabszewicz and Thisse (1986)). Here, however, our focus is on the vertical division of firm activities (in contrast to horizontal division). There also exists many papers which examine optimal location of multiple units having input-output linkages (for a survey, see, for example, Hansen et. al. (1987)). Here, our focus is on the general equilibrium aspects of cities with many firms having multiple units.

2. There currently exists a large body of literature on information technologies and their impacts on spatial and industrial organizations, which forms the background of this paper [for recent studies, see, for example, Estabrooks and Lamarche (1987) and Giaoutzi and Nijkamp (1988)]. Our purpose here is to develop a formal model for the study of interactions between the communication technologies and urban spatial organization.

3. Although back-units may interact with other agents (in the city as well as outside the city), such interaction costs are assumed to be the same regardless of their location in the city and hence these costs are suppressed in our model.

4. Note that all firms are assumed to be identical in their location behavior. They are, of course, different in other aspects such as the information or services they produce. Otherwise, no communication would be necessary among firms. 
5. It is true that communications are two-way activities. Recall, however, that all firms are assumed to be identical in terms of their location behavior. Therefore, for any given pair of front-units, the optimal level of contact between the two units (which is chosen independently by each) become the same. Hence, no problem of asymmetric demand in contact arises. Here, we assume implicitly that each pair of front-units splits equally their contact costs.

6. Here, it is assumed that the contact activity $q(x, z)$ contributes to the enhancement of the (physical) productivity of the firm and also to the increase in the demand for the product of the firm. Hence, $V[q(x, z)]$ represents the total contribution of $q(x, z)$ to the increase in the revenue of the firm. In equation (2.3), the benefits and costs of all activities of the firm other than those of communication, land and labor are assumed to be fixed and suppressed.

7. Notice that (2.3) represents a typical communication model (or endogenous contact model), while (2.6) a spatial externality model (or accessibility model). As we have seen, if we relate the accessibility function $a$ and benefit function $V$ by (2.5), the two models become mathematically equivalent. For further elaboration of this point, see Fujita and Smith (1990).

8. This complication does not arise for households. Although each household must choose two locations, a residential location and working location, it consumes land at the residential location only.

9. More generally, in (3.3) we can replace $P(y)$ by $P_{b}(y)$ and in (3.4) we can replace $P(x)$ by $P_{f}(x)$, where $P_{b}(y)$ and $P_{f}(x)$ can be any location of $y$ and $x$ respectively.

10. Generalizing (3.3) and (3.4), let us consider the following pair of decentralized profit functions:

$$
\begin{aligned}
& \pi_{f}(x \mid y)=A(x)-R(x) S_{f}-W(x) L_{f}-\alpha \Gamma(x, y)-P(y) \\
& \pi_{b}(y \mid x)=P(x)-R(y) S_{b}-W(y) L_{b}-\beta \Gamma(x, y) .
\end{aligned}
$$

Then, it can be readily verified that condition (3.1) can be satisfied if and only if $\alpha=$ $1=\beta$.

11. As noted before, $P(x)$ can be any function of $x$. However, in the following analysis we treat it as an unknown function because we wish to choose such a function $P(x)$ that is most convenient for the economic interpretation of equilibrium conditions.

12. The reason for the choice of notation $\pi_{f}(y)$ and $\pi_{b}(x)$ is as follows. Consider the frontunit and back-unit of any specific firm. Given the back-unit location, say $y$, the frontunit is free to choose its location. Therefore, the equilibrium (i.e., maximum) profit $\pi_{f}$ of that front-unit should be a function of $y$ (i.e., the location of the associated backunit); hence we denote it by $\pi_{f}(y)$. Similarly, given the front-unit location, say $x$, the back-unit is free to choose its location. Therefore, the equilibrium profit $\pi_{b}$ of that back-unit should be a function of $x$ (i.e., the location of the associated front-unit); hence we denote it by $\pi_{b}(x)$.

13. It is not difficult to show that we can always find $P^{*}$ such that condition (4.37) is satisfied for all $x \in f_{+}^{*}$. For examples of such $P^{*}$, see section 5 .

14. One way to derive the linear accessibility measure (4.41) is as follows. In the profit function (2.3), assume that the communication benefit function $V$ is given by the 
following entropy-type function:

$$
V(q)= \begin{cases}\frac{q}{\gamma}(1+\log \beta)-\frac{q}{\gamma} \log \frac{q}{\gamma} & \text { for } q<\gamma \beta \\ \beta & \text { for } q \geq \gamma \beta\end{cases}
$$

Then, solving the maximization problem (2.4), we obtain a negative exponential contact function, $q^{*}(c)=\gamma \beta e^{-\gamma c}$. Substituting this relation into (2.5), we have a negative exponential accessibility function, $a(c)=\beta e^{-\gamma c}$. Therefore, if we assume the following communication cost function,

$$
c(x, y)=\log \left(1-\frac{\gamma}{\beta}|x-y|\right)^{-1 / \gamma},
$$

then we obtain the linear accessibility measure (4.41). Notice that the communication cost function $c(x, y)$ above is convex in distance $|x-y|$, which is a realistic specification for the case of a face-to-face communication under consideration. For further explanations, see Fujita and Smith (1990).

15. More precisely, given each set of parameters, there exists one and only one Nash equilibrium land use pattern. However, given a Nash equilibrium land use pattern (e. g., Pattern $\mathrm{C}$ below), there can possibly exist a continuum of different equilibrium land rent curves which supports that land use pattern. This nonuniqueness in equilibrium land rent curve is not trivial because it implies nonuniqueness in equilibrium profitlevels of firms (or utility-levels of households): that is, in a Nash equilibrium (e.g., Pattern $\mathrm{C}$ below), firms at different locations may attain different levels of (total) profit.

16. About nonuniqueness of Nash equilibrium, we plan to discuss systematically in a subsequent paper.

17. This is because each household consumes a fixed amount of land $\left(S_{h}\right)$, and each firm has a fixed-coefficient technology.

18. We can interpret (5.2) as follows. Although the number of firms in the city is fixed (at $M$ ), households are assumed to be able to move at no cost between the city and the rest of the national economy. Then, in equilibrium, the total number of households in the city is given by $(2.8)$, and the utility of residents equals the national utility level given by (5.2).

19. In a manner similar to Ogawa and Fujita (1980), we can show that any (global) equilibrium configuration is symmetric in respect to the center of the city.

20. Actually, there exists infinitely many commuting correspondences which are consistent with Pattern C. Equation (5.7) represents one such example.

21. Notice that since the equilibrium rent curve $R^{*}(x)$ is defined by (4.17), the only unknowns left are $\pi_{f}^{*}, \pi_{b}^{*}$, and $P^{*}(x)$. It turns out that three unknowns cannot be determined uniquely. That is, given any $\pi_{f}^{*}$ and $\pi_{0}^{*}$ that satisfy (5.25), if we define $P^{*}(x)$ by (5.23) and (5.24), then all equilibrium conditions, (4.17) through (4.29), are satisfied by the configuration (4.5) determined above. This indeterminacy of the three unknowns can also be seen by (5.13) and (5.14). In the front-unit bid rent function (5. 13), what matters is not the individual values of $P^{*}(y)$ and $\pi_{f}^{*}$, but the sum, $P^{*}(y)+\pi_{f}^{*}$. 
Similarly, in (5.14), only the difference, $P^{*}(x)-\pi_{b}^{*}$, matters.

22. Again, correspondences (5.44) and (5.45) are just examples; there are infinitely many correspondences which are consistent with Pattern G. The same note applies to the correspondence (5.46).

23. It turns out that if there exists a fixed cost of intrafirm communications (i.e., $K>0$ ), then this statement does not hold true; that is, there exist global equilibria that cannot be subsisted as competitive equilibria. We plan to investigate systematically in a subsequent paper the relationship between global equilibria, Nash equilibria and competitive equilibria.

\section{References}

Estarbrooks, M. F. and R. H. Lamarche (eds.), 1987, Telecommunications: A strategic Perspective on Regional, Economic and Business Development, Canadian Institute for Research on Regional Development, Moncton.

Fujita, M. and H. Ogawa, 1982, Multiple equilibria and structural transition of non-monocentric urban configurations, Regional Science and Urban Economics, 12, 161-196.

Fujita, M. and T. Smith, 1990, Additive-interaction models of spatial agglomeration, Journal of Regional Science, 30, 51-74.

Gabszewicz, J. J. and J. F. Thisse, 1986, Spatial competition and the location of firms, in J. J. Gabszewicz, J. F. Thisse, M. Fujita, and U. Schweizer, Location Theory, Chur, Switzerland: Harwood Academic Publishes.

Giaoutzi, M. and P. Nijkamp (eds.), 1988, Information and Regional Development, Gower Publishing Company, Brookfield, Vermont.

Hansen, P., M. Labbé, D. Peeters, J.-F. Thisse and J. V. Henderson, 1987, Systems of Cities and Facility Location, Harwood Academic Publishers, New York.

Ogawa, H. and M. Fujita, 1980, Equilibrium land use in a nonmonocentric city, Joumal of Regional Science, $20,455-475$.

Ota, M. and M. Fujita, 1990, Communication Technologies and Spatial Organization of Multi-unit Firms in Metropolitan Areas, Working Paper 142 in Regional Science, Dept. of Regional Science, University of Pennsylvania, Philadelphia. 